\title{
Solving Reflexive Domain Equations in a Category of Complete Metric Spaces
}

\author{
Pierre America \\ Philips Research Laboratories \\ P.O. Box $80.000,5600$ JA Eindhoven, The Netherlands \\ Jan Rutten \\ Centre for Mathematics and Computer Science \\ P.O. Box 4079, 1009 AB Amsterdam, The Netherlands
}

\begin{abstract}
This paper presents a technique by which solutions to reflexive domain equations can be found in a certain category of complete metric spaces. The objects in this category are the (non-empty) metric spaces and the arrows consist of two maps: an isometric embedding and a non-distance-increasing left inverse to it. The solution of the equation is constructed as a fixed point of a functor over this category associated with the equation. The fixed point obtained is the direct limit (colimit) of a convergent tower. This construction works if the functor is contracting, which roughly amounts to the condition that it maps every embedding to an even denser one. We also present two additional conditions, each of which is sufficient to ensure that the functor has a unique fixed point (up to isomorphism). Finally, for a large class of functors, including function space constructions, we show that these conditions are satisfied, so that they are guaranteed to have a unique fixed point. The techniques we use are so reminiscent of Banach's fixed-point theorem that we feel justified to speak of a category-theoretic version of it.
\end{abstract}

1980 Mathematical Subject classification: 68B10,68C01.

1986 Computing Reviews Categories: D.1.3, D.3.1, F.3.2.

Key words and phrases: domain equations, complete metric spaces, category theory, converging towers, contracting functors, Banach's fixed-point theorem.

Note: This work was carried out in the context of ESPRIT project 415: Parallel Architectures and Languages for Advanced Information Processing - a VLSI-directed approach.

\section{INTRODUCTION}

The framework of complete metric spaces has proved to be very useful for giving a denotational semantics to programming languages, especially concurrent ones. For example, in the approach of De Bakker and Zucker [BZ] a process is modelled as the element of a suitable metric space, where the distance between two processes is defined in such a way that the smaller this distance is, the longer it takes before the two processes show a different behaviour. 
In order to construct a suitable metric space in which processes are to reside, we must solve a reflexive domain equation. For example, a simple language, where a process is a fixed sequence of uninterpreted atomic actions, gives rise to the equation

$$
P \cong\left\{p_{0}\right\} \bar{\cup}(A \times P)
$$

(Here $\bar{U}$ denotes the disjoint union operation.) In [BZ] an elementary technique was developed to solve such equations. Roughly, this consisted of starting with a small metric space, enriching it iteratively, and taking the metric completion of the union of all the obtained spaces.

In many cases this technique is sufficient to solve the equation at hand, but there are equations for which it does not work: equations where the domain variable $P$ occurs in the left-hand side of a function space construction, e.g.,

$$
P \cong\left\{p_{0}\right\} \bar{\cup}(P \rightarrow P) \text {. }
$$

This kind of equation arises when the semantic description is based on continuations (see for example [ABKR]). In this paper we present a technique by which these cases can also be solved, at least when we restrict the function space at hand to the non-distance-increasing functions.

The structure of this report is as follows: In section 2 we list some mathematical preliminaries. In section 3 we introduce our category $e$ of complete metric spaces, we define the concepts of converging tower and contracting functor. We show that a converging tower has a direct limit and that a contracting functor preserves such a limit. Then we see how a contracting functor gives rise to a converging tower and that the limit of this tower is a fixed point of the functor.

Section 4 presents two cases in which we can show that the fixed point we construct is the unique fixed point (up to isomorphism) of the contracting functor at hand. One case arises when we work in a base-point category: a category where every space has a specially designated basepoint and where every map preserves this base-point. The other case is where the functor is not only contracting, but also hom-contracting: it is a contraction on every function space.

Finally, in section 5, we present a large class of functors (including most of the ones we are interested in), for which we can show that each of them has a unique fixed point.

\section{Acknowledgements}

We would like to thank Jaco de Bakker, Frank de Boer, Joost Kok, Frank van der Linden, JohnJules Meyer and Erik de Vink for useful discussions on the contents of this paper. We are also grateful to Marino Delusso and Eline Meijs, who have typed this report. 


\section{MATHEMATICAL. PRELIMINARIES}

In this section we collect some definitions and properties concerning metric spaces, in order to refresh the reader's memory or to introduce him to this subject.

\subsection{Metric spaces}

Definirion 2.1 (Metric space)

A metric space is a pair $(M, d)$ with $M$ a non-empty set and $d$ a mapping $d: M \times M \rightarrow[0,1]$ (a metric or distance), which satisfies the following properties:

(a) $\forall x, y \in M[d(x, y)=0 \Leftrightarrow x=y]$

(b) $\forall x, y \in M[d(x, y)=d(y, x)]$

(c) $\forall x, y, z \in M[d(x, y) \leqslant d(x, z)+d(z, y)]$.

We call $(M, d)$ an ultra-metric space if the following stronger version of property (c) is satisfied:

(c') $\forall x, y, z \in M[d(x, y) \leqslant \max \{d(x, z), d(z, y)\}]$.

Note that we consider only metric spaces with bounded diameter: the distance between two points never exceeds 1 .

Example

Let $\mathrm{A}$ be an arbitrary set. The discrete metric $d_{A}$ on $A$ is defined as follows. Let $x, y \in A$, then

$$
d_{A}(x, y)= \begin{cases}0 & \text { if } x=y \\ 1 & \text { if } x \neq y .\end{cases}
$$

\section{DEFINITION 2.2}

Let $(M, d)$ be a metric space, let $\left(x_{i}\right)_{i}$ be a sequence in $M$.

(a) We say that $\left(x_{i}\right)_{i}$ is a Cauchy sequence whenever we have: $\forall \epsilon>0 \quad \exists N \in \mathbb{N} \forall n, m>N\left[d\left(x_{n}, x_{m}\right)<\epsilon\right]$.

(b) Let $x \in M$. We say that $\left(x_{i}\right)_{i}$ converges to $x$ and call $x$ the limit of $\left(x_{i}\right)_{i}$ whenever we have: $\forall \epsilon>0 \quad \exists N \in \mathbb{N} \forall n>N\left[d\left(x, x_{n}\right)<\epsilon\right]$.

Such a sequence we call convergent. Notation: $\lim _{i \rightarrow \infty} x_{i}=x$.

(c) The metric space $(M, d)$ is called complete whenever each Cauchy sequence converges to an element of $M$.

\section{Definition 2.3}

Let $\left(M_{1}, d_{1}\right),\left(M_{2}, d_{2}\right)$ be metric spaces.

(a) We say that $\left(M_{1}, d_{1}\right)$ and $\left(M_{2}, d_{2}\right)$ are isometric if there exists a bijection $f: M_{1} \rightarrow M_{2}$ such that:

$\forall x, y \in M_{1}\left[d_{2}(f(x), f(y))=d_{1}(x, y)\right]$. We then write $M_{1} \cong M_{2}$. When $f$ is not a bijection (but only an injection), we call it an isometric embedding. 
(b) Let $f: M_{1} \rightarrow M_{2}$ be a function. We call $f$ continuous whenever for each sequence $\left(x_{i}\right)_{i}$ with limit $x$ in $M_{1}$ we have that $\lim _{i \rightarrow \infty} f\left(x_{i}\right)=f(x)$.

(c) Let $A \geqslant 0$. With $M_{1} \rightarrow^{A} M_{2}$ we denote the set of functions $f$ from $M_{1}$ to $M_{2}$ that satisfy the following property:

$\forall x, y \in M_{1}\left[d_{2}(f(x), f(y)) \leqslant A \cdot d_{1}(x, y)\right]$.

Functions $f$ in $M_{1} \rightarrow{ }^{1} M_{2}$ we call non-distance-increasing (NDI), functions $f$ in $M_{1} \rightarrow{ }^{\epsilon} M_{2}$ with $0 \leqslant \epsilon<1$ we call contracting.

\section{Proposition 2.4}

(a) Let $\left(M_{1}, d_{1}\right),\left(M_{2}, d_{2}\right)$ be metric spaces. For every $A \geqslant 0$ and $f \in M_{1} \rightarrow{ }^{A} M_{2}$ we have: $f$ is continuous.

(b) (Banach's fixed-point theorem)

Let $(M, d)$ be a complete metric space and $f: M \rightarrow M$ a contracting function. Then there exists an $x \in M$ such that the following holds:

(1) $f(x)=x$ ( $x$ is a fixed point of $f)$,

(2) $\forall y \in M[f(y)=y \Rightarrow y=x]$ ( $x$ is unique),

(3) $\forall x_{0} \in M\left[\lim _{n \rightarrow \infty} f^{(n)}\left(x_{0}\right)=x\right]$, where $f^{(n+1)}\left(x_{0}\right)=f\left(f^{(n)}\left(x_{0}\right)\right)$ and $f^{(0)}\left(x_{0}\right)=x_{0}$.

DefinITION 2.5 (Closed subsets)

A subset $X$ of a complete metric space $(M, d)$ is called closed whenever each Cauchy sequence in $X$ converges to an element of $X$.

\section{DEFINITION 2.6}

Let $(M, d),\left(M_{1}, d_{1}\right), \ldots,\left(M_{n}, d_{n}\right)$ be metric spaces.

(a) With $M_{1} \rightarrow M_{2}$ we denote the set of all continuous functions from $M_{1}$ to $M_{2}$. We define a metric $d_{F}$ on $M_{1} \rightarrow M_{2}$ as follows. For every $f_{1}, f_{2} \in M_{1} \rightarrow M_{2}$

$$
d_{F}\left(f_{1}, f_{2}\right)=\sup _{x \in M_{1}}\left\{d_{2}\left(f_{1}(x), f_{2}(x)\right)\right\} .
$$

For $A \geqslant 0$ the set $M_{1} \rightarrow{ }^{A} M_{2}$ is a subset of $M_{1} \rightarrow M_{2}$, and a metric on $M_{1} \rightarrow{ }^{A} M_{2}$ can be obtained by taking the restriction of the corresponding $d_{F}$.

(b) With $M_{1} \bar{\cup} \ldots \bar{\cup} M_{n}$ we denote the disjoint union of $M_{1}, \ldots, M_{n}$, which can be defined as $\{1\} \times M_{1} \cup \cdots \cup\{n\} \times M_{n}$. We define a metric $d_{U}$ on $M_{1} \bar{\cup} \cdots \bar{\cup} M_{n}$ as follows. For every $x, y \in M_{\cdot 1} \bar{\cup} \cdots \bar{\cup} M_{n}$

$$
d_{U}(x, y)= \begin{cases}d_{j}(x, y) & \text { if } x, y \in\{j\} \times M_{j}, 1 \leqslant j \leqslant n \\ 1 & \text { otherwise. }\end{cases}
$$

(c) We define a metric $d_{P}$ on $M_{1} \times \cdots \times M_{n}$ by the following clause.

For every $\left(x_{1}, \ldots, x_{n}\right),\left(y_{1}, \ldots, y_{n}\right) \in M_{1} \times \cdots \times M_{n}$

$$
d_{P}\left(\left(x_{1}, \ldots, x_{n}\right),\left(y_{1}, \ldots, y_{n}\right)\right)=\max _{i}\left\{d_{i}\left(x_{i}, y_{i}\right)\right\} .
$$


(d) Let $\mathscr{P}_{c l}(M)=\operatorname{def}\{X|X \subseteq M| X$ is closed and non-empty $\}$. We define a metric $d_{H}$ on $\mathscr{P}_{c l}(M)$, called the Hausdorff distance, as follows. For every $X, Y \in \mathscr{P}_{c l}(M)$

$$
d_{H}(X, Y)=\max \left\{\sup _{x \in X}\{d(x, Y)\}, \sup _{y \in Y}\{d(y, X)\}\right\},
$$

where $d(x, Z)=\operatorname{def}_{\inf } \in Z\{d(x, z)\}$ for every $Z \subseteq M, x \in M$.

An equivalent definition would be to set $V_{r}(X)=\{y \in M \mid \exists x \in X[d(x, y)<r]\}$ for $r>0, X \subset M$, and then to define

$$
d_{H}(X, Y)=\inf \left\{r>0 \mid X \subset V_{r}(Y) \wedge Y \subset V_{r}(X)\right\}
$$

\section{Proposition 2.7}

Let $(M, d),\left(M_{1}, d_{1}\right), \ldots,\left(M_{n}, d_{n}\right), d_{F}, d_{U}, d_{P}$ and $d_{H}$ be as in definition 2.6 and suppose that $(M, d),\left(M_{1}, d_{1}\right), \ldots,\left(M_{n}, d_{n}\right)$ are complete. We have that

(a) $\left(M_{1} \rightarrow M_{2}, d_{F}\right),\left(M_{1} \rightarrow{ }^{A} M_{2}, d_{F}\right)$,

(b) $\left(M_{1} \bar{\cup} \cdots \bar{\cup} M_{n}, d_{U}\right)$,

(c) $\left(M_{1} \times \cdots \times M_{n}, d_{P}\right)$,

(d) $\left(\rho_{c l}(M), d_{H}\right)$

are complete metric spaces. If $(M, d)$ and $\left(M_{i}, d_{i}\right)$ are all ultra-metric spaces these composed spaces are again ultra-metric. (Strictly spoken, for the completeness of $M_{1} \rightarrow M_{2}$ and $M_{1} \rightarrow{ }^{A} M_{2}$ we do not need the completeness of $M_{1}$. The same holds for the ultra-metric property.)

If in the sequel we write $M_{1} \rightarrow M_{2}, M_{1} \rightarrow{ }^{A} M_{2}, M_{1} \bar{\cup} \cdots \bar{\cup} M_{n}, M_{1} \times \cdots \times M_{n}$ or $\mathscr{P}_{c l}(M)$, we mean the metric space with the metric defined above.

The proofs of proposition 2.7 (a), (b) and (c) are straightforward. Part (d) is more involved. It can be proved with the help of the following characterization of the completeness of $\left(\odot_{c l}(M), d_{H}\right)$.

\section{PROPOSITION 2.8}

Let $\left(\mathscr{P}_{c l}(M), d_{H}\right)$ be as in definition 2.6. Let $\left(X_{i}\right)_{i}$ be a Cauchy sequence in $\mathscr{P}_{c l}(M)$. We have:

$$
\lim _{i \rightarrow \infty} X_{i}=\left\{\lim _{i \rightarrow \infty} x_{i} \mid x_{i} \in X_{i},\left(x_{i}\right)_{i} \text { a Cauchy sequence in } M\right\} .
$$

Proofs of proposition 2.7(d) and 2.8 can be found in (for instance) [Du] and [En]. Proposition 2.8 is due to Hahn [Ha]. The proofs are also repeated in [BZ].

THEOREM 2.9 (Metric completion)

Let $M$ be an arbitrary metric space. Then there exists a metric space $\bar{M}$ (called the completion of $M$ ) together with an isometric embedding $i: M \rightarrow \bar{M}$ such that:

(1) $\bar{M}$ is complete

(2) For every complete metric space $M^{\prime}$ and isometric embedding $j: M \rightarrow M^{\prime}$ there exists a unique isometric embedding $\bar{j}: \bar{M} \rightarrow M^{\prime}$ such that $\bar{j} \circ i=j$. 


\section{PROOF}

The space $\bar{M}$ is constructed by taking the set of all Cauchy sequences in $M$ and dividing it out by the equivalence relation $\equiv$ defined by

$$
\left(x_{n}\right)_{n} \equiv\left(y_{n}\right)_{n}={ }^{\text {def }} \lim _{n \rightarrow \infty} d\left(x_{n}, y_{n}\right)=0 .
$$

The metric $d_{c}$ on $\bar{M}$ is defined by

$$
d_{c}\left(\left[\left(x_{n}\right)\right] \equiv,\left[\left(y_{n}\right)\right] \equiv\right)={ }^{\operatorname{def}} \lim _{n \rightarrow \infty} d\left(x_{n}, y_{n}\right)
$$

and the embedding $i$ will map every $x \in M$ to the equivalence class of the sequence of which all elements are equal to $x$ :

$$
i(x)=\left[(x)_{n}\right] \equiv .
$$

It is easy to show that $\bar{M}$ and $i$ satisfy the above properties.

\section{A CATEGORY OF COMPLETE METRIC SPACES}

In this section we want to generalize the technique of solving reflexive domain equations of $\mathrm{De}$ Bakker and Zucker ([BZ]). We shall first give an example of their approach and then explain how it can be extended.

Consider a domain equation

$$
P \cong\left\{p_{0}\right\} \bar{\cup}(A \times P),
$$

with $A$ an arbitrary set. In [BZ] a complete metric space that satisfies this equation is constructed as follows. An increasing sequence $A^{(0)} \subseteq A^{(1)} \subseteq \cdots$ of metric spaces is defined by

(0) $A^{(0)}=\left\{p_{0}\right\}, d_{0}$ trivial,

$$
\begin{aligned}
& (n+1) A^{(n+1)}=\left\{p_{0}\right\} \cup A \times A^{(n)}, \\
& d_{n+1}\left(p_{0}, q\right)=1 \text { if } q \in A^{(n+1)}, q \neq p_{0}, \\
& d_{n+1}\left(<a_{1}, p_{1}>,<a_{2}, p_{2}>\right)=\left\{\begin{array}{cc}
1 & \text { if } a_{1} \neq a_{2} \\
\frac{1}{2} \cdot d_{n}\left(p_{1}, p_{2}\right) & \text { if } a_{1}=a_{2} .
\end{array}\right.
\end{aligned}
$$

Note that for every $i \geqslant 0, A^{(i)}$ is a subspace of $A^{(i+1)}$. Their union is defined as

$$
A^{\star}=\bigcup_{n \in \mathbb{N}} A^{(n)}
$$

and a domain $A^{\infty}$ is defined as the metric completion of this union: 


$$
A^{\infty}=\overline{A^{\star}} \text {. }
$$

It is then proved that $A^{\infty}$ satisfies the equation. (We observe that $A^{\star}$ is isometric to the set of all finite sequences of elements of $A$, while $A^{\infty}$ is isometric to the set of all finite and infinite sequences, in both cases with a suitable metric.)

In order to extend this approach, we shall formulate a number of category-theoretic generalizations of some of the concepts used in the construction described above.

First we shall define a converging tower to be the counterpart of an increasing sequence of metric spaces; then the construction of a direct limit of such a tower will be the generalization of the metric completion of the union of such a sequence. Finally we shall give a generalized version of Banach's fixed-point theorem.

For this purpose we define a category $\mathcal{C}$ of complete metric spaces.

Definitron 3.1 (Category of complete metric spaces)

Let $\mathcal{C}$ denote the category that has complete metric spaces for its objects. The arrows $\iota$ in $\mathcal{C}$ are defined as follows. Let $M_{1}, M_{2}$ be complete metric spaces. Then $M_{1} \rightarrow^{\imath} M_{2}$ denotes a pair of maps $M_{1} \underset{j}{\stackrel{i}{\rightleftarrows}} M_{2}$, satisfying the following properties:

(a) $i$ is an isometric embedding,

(b) $j$ is non-distance-increasing (NDI),

(c) $j \circ i=i d_{M_{1}}$.

(We sometimes write $\langle i, j\rangle$ for $\iota$.) Composition of the arrows is defined in the obvious way.

\section{REMARK}

For the basic definitions from category theory we refer the reader to [ML].

We can consider $M_{1}$ as an approximation of $M_{2}$ : in a sense the set $M_{2}$ contains more information than $M_{1}$, because $M_{1}$ can be isometrically embedded into $M_{2}$. Elements in $M_{2}$ are approximated by elements in $M_{1}$. For an element $m_{2} \in M_{2}$ its (best) approximation in $M_{1}$ is given by $j\left(m_{2}\right)$. (The reason why $j$ should be NDI is, at this point, difficult to motivate.)

When we informally rephrase clause (c), it states that the approximation in $M_{1}$ of the embedding of an element $m_{1} \in M_{1}$ into $M_{2}$ is again $m_{1}$. Or, in other words, that $M_{2}$ is a consistent extension of $M_{1}$.

\section{Definition 3.2}

For every arrow $M_{1} \rightarrow^{\imath} M_{2}$ in $e$ with $\iota=<i, j>$ we define

$$
\delta(\iota)=d_{M_{2} \rightarrow M_{2}}\left(i \circ j, i d_{M_{2}}\right)\left(=\sup _{m_{2} \in M_{2}}\left\{d_{M_{2}}\left(i \circ j\left(m_{2}\right), m_{2}\right)\right\}\right) .
$$


This number plays an important role in our theory. It can be regarded as a measure of the quality with which $M_{2}$ is approximated by $M_{1}$ : the smaller $\delta(\iota)$, the denser $M_{1}$ is embedded into $M_{2}$. We next try to formalize a generalization of increasing sequences of metric spaces by the following definition.

Definition 3.3 (Converging tower)

(a) We call a sequence $\left(D_{n}, \iota_{n}\right)_{n}$ of complete metric spaces and arrows a tower whenever we have that

$\forall n \in \mathbb{N}\left[D_{n} \rightarrow^{\iota_{n}} D_{n+1} \in \mathcal{C}\right]$

$$
D_{0} \rightarrow{ }^{l_{0}} D_{1} \rightarrow^{l_{1}} D_{2} \rightarrow \cdots \rightarrow D_{n} \rightarrow{ }^{l_{n}} D_{n+1} \rightarrow \cdots
$$

(b) The sequence $\left(D_{n}, \iota_{n}\right)_{n}$ is called a converging tower when furthermore the following condition is satisfied:

$\forall \epsilon>0 \exists N \in \mathbb{N} \forall m>n \geqslant N\left[\delta\left(\iota_{n m}\right)<\epsilon\right]$, where $\iota_{n m}=\iota_{m-1} \circ \cdots \iota_{n}: D_{n} \rightarrow D_{m}$.

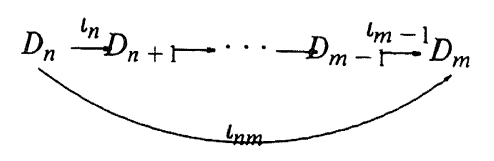

\section{EXAMPLE 3.4}

A special case of a converging tower is a sequence $\left(D_{n}, l_{n}\right)_{n}$ that satisfies the following conditions:

(a) $\forall n \in \mathbb{N}\left[D_{n} \rightarrow^{\iota_{n}} D_{n+1} \in \bigodot\right]$,

(b) $\exists \epsilon\left[0 \leqslant \epsilon<1 \wedge \forall n \in \mathbb{N}\left[\delta\left(\iota_{n}+1\right) \leqslant \epsilon \cdot \delta\left(\iota_{n}\right)\right]\right]$.

(Note that $\delta\left(\iota_{n m}\right) \leqslant \delta\left(\iota_{n}\right)+\cdots+\delta\left(\iota_{m-1}\right) \leqslant \epsilon^{n} \cdot \delta\left(\iota_{0}\right)+\cdots+\epsilon^{m-1} \cdot \delta\left(\iota_{0}\right) \leqslant \frac{\epsilon^{n}}{1-\epsilon} \cdot \delta\left(\iota_{0}\right)$.)

\section{EXAMPLE 3.5}

Let $A^{(0)} \subseteq A^{(1)} \subseteq \cdots$ be the sequence of metric spaces defined at the beginning of this chapter. We show how it can be transformed into a converging tower, by defining a sequence of arrows $\left(\iota_{n}\right)_{n}$ (with $\iota_{n}=<i_{n}, j_{n}>$ ) with induction on $n$ :

(0) $i_{0}\left(p_{0}\right)=p_{0}, j_{0}$ trivial,

$$
\begin{aligned}
(n+1) & i_{n+1}: A^{(n+1)} \rightarrow A^{(n+2)}, \operatorname{trivial}\left(i_{n+1}(p)=p\right), \\
& j_{n+1}: A^{(n+2)} \rightarrow A^{(n+1)}, \\
& j_{n+1}\left(p_{0}\right)=p_{0}, \\
& j_{n+1}(<a, p>)=<a, j_{n}(p)>\text { for }<a, p>\in A^{(n+2)} .
\end{aligned}
$$

It is not difficult to see that we have obtained a tower 


$$
A^{(0)} \rightarrow^{l_{0}} A^{(1)} \rightarrow^{l_{1}} \cdots
$$

which is converging.

\subsection{The direct limit construction}

In this subsection we show that in our category $e$ every converging tower has an initial cone. The construction of such an initial cone for a given tower (the direct limit construction) generalizes the technique of forming the metric completion of the union of an increasing sequence of metric spaces.

Before we treat the inverse limit construction, we first give the definition of a cone and an initial cone and then formulate a criterion for the initiality of a cone.

\section{Definition 3.6 (Cone)}

Let $\left(D_{n}, l_{n}\right)_{n}$ be a tower. Let $D$ be a complete metric space and $\left(\gamma_{n}\right)_{n}$ a sequence of arrows. We call $\left(D,\left(\gamma_{n}\right)_{n}\right)$ a cone for $\left(D_{n}, l_{n}\right)_{n}$ whenever the following condition holds:

$$
\begin{aligned}
& \forall n \in \mathbb{N}\left[D_{n} \rightarrow^{\gamma_{n}} D \in \circlearrowright \wedge \gamma_{n}=\gamma_{n}+1^{\circ} \iota_{n}\right] .
\end{aligned}
$$

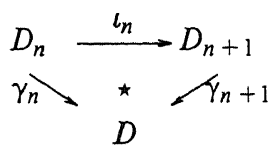

DefrNITION 3.7 (Initial cone)

A cone $\left(D,\left(\gamma_{n}\right)_{n}\right)$ of a tower $\left(D_{n}, l_{n}\right)_{n}$ is called initial whenever for every other cone $\left(D^{\prime},\left(\gamma_{n}^{\prime}\right)_{n}\right)$ of $\left(D_{n}, \iota_{n}\right)_{n}$ there exists a unique arrow $\iota: D \rightarrow D^{\prime}$ in $e$ such that:

$$
\forall n \in \mathbb{N}\left[\left\llcorner\circ \gamma_{n}=\gamma_{n}^{\prime}\right]\right.
$$

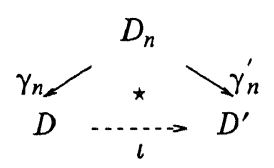

LEMMA 3.8 (Initiality lemma)

Let $\left(D_{n}, l_{n}\right)_{n}$ be a converging tower with a cone $\left(D,\left(\gamma_{n}\right)_{n}\right)$. Let $\gamma_{n}=\left\langle\alpha_{n}, \beta_{n}\right\rangle$. We have:

$D$ is an initial cone $\Leftrightarrow \lim _{n \rightarrow \infty} \alpha_{n} \circ \beta_{n}=i d_{D}$.

PROOF 
$\Leftarrow$

Suppose $\lim _{n \rightarrow \infty} \alpha_{n} \circ \beta_{n}=i d_{D}$. Let $\left(D^{\prime},\left(\gamma_{n}^{\prime}\right)_{n}\right)$, with $\gamma_{n}^{\prime}=\left\langle\alpha_{n}^{\prime}, \beta_{n}^{\prime}\right\rangle$, be another cone for $\left(D_{n}, \iota_{n}\right)_{n}$. We have to prove the existence of a unique arrow $D \rightarrow^{\prime} D^{\prime} \in \mathcal{C}$ such that

$$
\forall n \in \mathbb{N}\left[\iota \circ \gamma_{n}=\gamma_{n}^{\prime}\right]
$$

First we construct an embedding $i: D \rightarrow D^{\prime}$, then a projection $j: D^{\prime} \rightarrow D$. Next, the arrow $\iota$ will be defined as $\iota=<i, j>$.

For every $n \in \mathbb{N}$ we have

$$
\alpha_{n}^{\prime} \circ \beta_{n} \in D \rightarrow D^{\prime}
$$

We show that $\left(\alpha_{n}^{\prime} \circ \beta_{n}\right)_{n}$ is a Cauchy sequence in $D \rightarrow D^{\prime}$ and then use the completeness of this function space to define $i$ as the limit of that sequence.

Let $m>n \geqslant 0$. We have

$$
\begin{aligned}
& d_{D \rightarrow D^{\prime}}\left(\alpha_{m}^{\prime} \circ \beta_{m}, \alpha_{n}^{\prime} \circ \beta_{n}\right)= \\
& d_{D \rightarrow D^{\prime}}\left(\alpha_{m}^{\prime} \circ \beta_{m}, \alpha_{m}^{\prime} \circ i_{n m^{\circ} j_{n m}} \circ \beta_{m}\right)= \\
& \sup _{x \in D}\left\{d_{D^{\prime}}\left(\alpha_{m}^{\prime} \circ \beta_{m}(x), \alpha_{m}^{\prime} \circ i_{n m^{\circ}} \circ j_{n m} \circ \beta_{m}(x)\right)\right\}=
\end{aligned}
$$

[ because $\alpha_{m}^{\prime}$ is isometric ]

$$
\sup _{x \in D}\left\{d_{D_{m}}\left(\beta_{m}(x), i_{n m} \circ j_{n m} \circ \beta_{m}(x)\right)\right\}=
$$

[ because $\beta_{m}$ is surjective ]

$$
\begin{aligned}
& \sup _{x \in D_{m}}\left\{d_{D_{m}}\left(x, i_{n m} \circ j_{n m}(x)\right)\right\}= \\
& \left.d_{D_{m} \rightarrow D_{m}}\left(i d_{D_{m}}, i_{n m} \circ j_{n m}\right)\right)=\delta\left(\iota_{n m}\right) .
\end{aligned}
$$

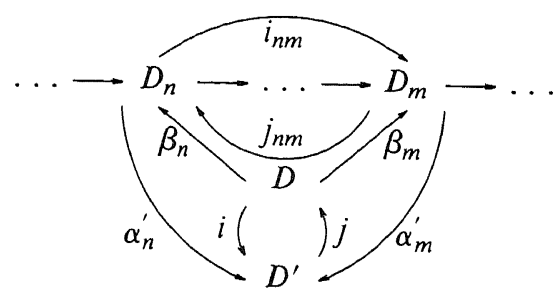

Let $\epsilon>0$. Because $\left(D_{n}, \iota_{n}\right)_{n}$ is a converging tower there is an $N \in \mathbb{N}$ such that

$$
\forall m>n \geqslant N\left[\delta\left(\iota_{n m}\right)<\epsilon\right] .
$$

Thus $\left(\alpha_{n}^{\prime} \circ \beta_{n}\right)_{n}$ is a Cauchy sequence. We define

$$
i=\lim _{n \rightarrow \infty} \alpha_{n}^{\prime} \circ \beta_{n} .
$$

We prove that $i$ is isometric by showing: 
$\forall x, y \in D\left[d_{D^{\prime}}(i(x), i(y))=d_{D}(x, y)\right]$

Let $x, y \in D$, we have

$$
\begin{aligned}
& d_{D^{\prime}}(i(x), i(y))= \\
& d_{D^{\prime}}\left(\lim _{n \rightarrow \infty} \alpha_{n}^{\prime} \circ \beta_{n}(x), \lim _{n \rightarrow \infty} \alpha_{n}^{\prime} \circ \beta_{n}(y)\right)= \\
& \lim _{n \rightarrow \infty} d_{D^{\prime}}\left(\alpha_{n}^{\prime} \circ \beta_{n}(x), \alpha_{n}^{\prime} \circ \beta_{n}(y)\right)= \\
& \text { [ because } \alpha_{n}^{\prime} \text { is isometric ] } \\
& \lim _{n \rightarrow \infty} d_{D_{n}}\left(\beta_{n}(x),\left(\beta_{n}(y)\right)=\right. \\
& {\left[\text { because } \alpha_{n}\right. \text { is isometric ] }} \\
& \lim _{n \rightarrow \infty} d_{D}\left(\alpha_{n} \circ \beta_{n}(x), \alpha_{n} \circ \beta_{n}(y)\right)= \\
& d_{D}\left(\lim _{n \rightarrow \infty} \alpha_{n} \circ \beta_{n}(x), \lim _{n \rightarrow \infty} \alpha_{n} \circ \beta_{n}(y)\right)= \\
& d_{D}(x, y) .
\end{aligned}
$$

Thus $i$ is isometric.

Similar to the definition of $i$ we choose

$$
j=\lim _{n \rightarrow \infty} \alpha_{n} \circ \beta_{n}^{\prime} .
$$

We have that $j$ is NDI, because, for $x, y \in D^{\prime}$ :

$$
\begin{aligned}
& d_{D}(j(x), j(y))= \\
& d_{D}\left(\lim _{n \rightarrow \infty} \alpha_{n} \circ \beta_{n}^{\prime}(x), \lim _{n \rightarrow \infty} \alpha_{n} \circ \beta_{n}^{\prime}(y)\right)= \\
& \lim _{n \rightarrow \infty} d_{D}\left(\alpha_{n} \circ \beta_{n}^{\prime}(x), \alpha_{n} \circ \beta_{n}^{\prime}(y)\right)= \\
& \text { [ because } \alpha_{n} \text { is isometric ] } \\
& \lim _{n \rightarrow \infty} d_{D_{n}}\left(\beta_{n}^{\prime}(x),\left(\beta_{n}^{\prime}(y)\right) \leqslant\right. \\
& \text { [ because } \beta_{n}^{\prime} \text { is NDI ] } \\
& \lim _{n \rightarrow \infty} d_{D^{\prime}}(x, y)= \\
& d_{D^{\prime}}(x, y) .
\end{aligned}
$$

We also show : $j \circ i=i d_{D}$. Let $x \in D$, then

$$
\begin{aligned}
& j \circ i(x)= \\
& j\left(\lim _{n \rightarrow \infty} \alpha_{n}^{\prime} \circ \beta_{n}(x)\right)= \\
& \lim _{n \rightarrow \infty} j \circ \alpha_{n}^{\prime} \circ \beta_{n}(x)=
\end{aligned}
$$




$$
\begin{aligned}
& \lim _{n \rightarrow \infty} \lim _{m \rightarrow \infty} \alpha_{m} \circ \beta_{m}^{\prime} \circ \alpha_{n}^{\prime} \circ \beta_{n}(x)= \\
& \lim _{n \rightarrow \infty} \alpha_{n} \circ \beta_{n}^{\prime} \circ \alpha_{n}^{\prime} \circ \beta_{n}(x)= \\
& {\left[\text { because } \beta_{n}^{\prime} \circ \alpha_{n}^{\prime}=i d_{D_{n}}\right]} \\
& \lim _{n \rightarrow \infty} \alpha_{n} \circ \beta_{n}(x)=x .
\end{aligned}
$$

Now we can define

$$
\iota=<i, j>,
$$

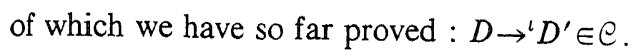

Next we have to verify that $\iota$ satisfies the condition

$$
\forall m \in \mathbb{N}\left[\iota^{\circ} \gamma_{m}=\gamma_{m}^{\prime}\right]
$$

This amounts to

$$
\forall m \in \mathbb{N}\left[i \circ \alpha_{m}=\alpha_{m}^{\prime} \wedge \beta_{m} \circ j=\beta_{m}^{\prime}\right] .
$$

Let $m \geqslant 0$. We only prove the first part of the conjunction. We have

$$
\begin{aligned}
i \circ \alpha_{m} & =\left(\lim _{n \rightarrow \infty} \alpha_{n}^{\prime} \circ \beta_{n}\right) \circ \alpha_{m} \\
& =\left(\lim _{n \rightarrow \infty} \alpha_{n+m}^{\prime} \circ \beta_{n+m}\right) \circ \alpha_{m} \\
& =\lim _{n \rightarrow \infty} \alpha_{n+m}^{\prime} \circ \beta_{n+m} \circ \alpha_{m} \\
& =\lim _{n \rightarrow \infty} \alpha_{n+m}^{\prime} \circ \beta_{n+m} \circ \alpha_{n+m} \circ i_{m, m+n} \\
& =\lim _{n \rightarrow \infty} \alpha_{n+m}^{\prime} \circ i d_{D_{n+m}} \circ i_{m, m+n} \\
& =\lim _{n \rightarrow \infty} \alpha_{m}^{\prime}=\alpha_{m}^{\prime} .
\end{aligned}
$$

Finally we show that $\iota$ is unique. Suppose $D \rightarrow \iota^{\prime} D^{\prime}$, with $\iota^{\prime}=\left\langle i^{\prime}, j^{\prime}\right\rangle$, is another arrow in $\varrho$, that satisfies

$$
\forall m \in \mathbb{N}\left[\iota^{\prime} \circ \gamma_{m}=\gamma_{m}^{\prime}\right] .
$$

We only show that $i^{\prime}=i$, leaving the proof of $j^{\prime}=j$ to the reader:

$$
\begin{aligned}
i^{\prime} & =i^{\prime} \circ i d_{D} \\
& =i^{\prime} \circ \lim _{m \rightarrow \infty} \alpha_{m} \circ \beta_{m} \\
& =\lim _{m \rightarrow \infty} i^{\prime} \circ \alpha_{m} \circ \beta_{m} \\
& =\lim _{m \rightarrow \infty} \alpha_{m}^{\prime} \circ \beta_{m} \\
& =i .
\end{aligned}
$$


$\Rightarrow$

Suppose now that $\left(D,\left(\gamma_{n}\right)_{n}\right)$ is an initial cone of the converging tower $\left(D_{n}, \iota_{n}\right)_{n}$. We have to prove that

$$
\lim _{m \rightarrow \infty} \alpha_{n} \circ \beta_{n}=i d_{D}
$$

By an argument similar to the proof for $\left(\alpha_{n}^{\prime} \circ \beta_{n}\right)_{n}$ above, we have that $\left(\alpha_{n} \circ \beta_{n}\right)_{n}$ is a Cauchy sequence. We define

$$
\begin{aligned}
& f=\lim _{n \rightarrow \infty} \alpha_{n} \circ \beta_{n}, \\
& D^{\prime}=\{x|x \in D| f(x)=x\} .
\end{aligned}
$$

We set out to prove that $D^{\prime}=D$.

The set $D^{\prime}$ is a closed subset of $D$, so it again constitutes a complete metric space. For each $n \in \mathbb{N}$ we have

$$
\alpha_{n}: D_{n} \rightarrow D^{\prime}
$$

because of the following argument. Let $d \in D_{n}$, then:

$$
\begin{aligned}
& f\left(\alpha_{n}(d)\right)= \\
& \lim _{m \rightarrow \infty} \alpha_{m} \circ \beta_{m}\left(\alpha_{n}(d)\right)= \\
& \lim _{m \rightarrow \infty} \alpha_{n+m} \circ \beta_{n+m} \circ\left(\alpha_{n}(d)\right)= \\
& \lim _{m \rightarrow \infty} \alpha_{n+m} \circ \beta_{n+m} \circ \alpha_{n+m} \circ i_{n, n+m}(d)= \\
& \lim _{m \rightarrow \infty} \alpha_{n+m} \circ i_{n, n+m}(d)= \\
& \lim _{m \rightarrow \infty} \alpha_{n}(d)= \\
& \alpha_{n}(d) .
\end{aligned}
$$

So $f\left(\alpha_{n}(d)\right)=\alpha_{n}(d)$, and thus $\alpha_{n}(d) \in D^{\prime}$.

Next we define, for each $n \in \mathbb{N}$ :

$$
\begin{aligned}
& \alpha_{n}^{\prime}=\alpha_{n}, \\
& \beta_{n}^{\prime}=\beta_{n} D^{\prime} \quad\left(\beta_{n} \text { restricted to } D^{\prime}\right), \\
& \gamma_{n}^{\prime}=\left\langle\alpha_{n}^{\prime}, \beta_{n}^{\prime}\right\rangle .
\end{aligned}
$$

It is clear that $\left(D^{\prime},\left(\gamma_{n}^{\prime}\right)_{n}\right)$ is another cone for $\left(D_{n}, \iota_{n}\right)_{n}$. Because $\left(D,\left(\gamma_{n}\right)_{n}\right)$ is initial, there exists a unique arrow $D \rightarrow{ }^{\iota_{1}} D^{\prime} \in \mathcal{C}$ with $\iota_{1}=\left\langle i_{1}, j_{1}\right\rangle$ such that

$$
\forall n \in \mathbb{N}\left[\iota_{1} \circ \gamma_{n}=\gamma_{n}^{\prime}\right]
$$

The set $D^{\prime}$ can also be embedded into $D$ : let $D^{\prime} \rightarrow^{\iota_{2}} D$, with $\iota_{2}=<i_{2}, j_{2}>$, be defined by 


$$
\begin{aligned}
& i_{2}=i d_{D^{\prime}}, \\
& j_{2}=i_{1} .
\end{aligned}
$$

Then $D^{\prime} \rightarrow{ }^{i_{2}} D \in e$. For $i_{2}$ is isometric, $j_{2}$ is NDI and the following argument shows that $j_{2} \circ i_{2}=i d_{D^{\prime}}$. Let $d \in D^{\prime}$. Then

$$
\begin{aligned}
& j_{2} \circ i_{2}(d)=j_{2}(d) \\
& =i_{1}(d) \\
& =\left[\text { because } d \in D^{\prime} \text {, we have } f(d)=d\right. \text {; } \\
& \text { in other words, }\left(\lim _{n \rightarrow \infty} \alpha_{n} \circ \beta_{n}\right)(d)=d \text { ] } \\
& \left(i_{1} \circ\left(\lim _{n \rightarrow \infty} \alpha_{n} \circ \beta_{n}\right)\right)(d) \\
& =\lim _{n \rightarrow \infty}\left(i_{1} \circ \alpha_{n} \circ \beta_{n}\right)(d) \\
& =\lim _{n \rightarrow \infty}\left(\alpha_{n}^{\prime} \circ \beta_{n}\right)(d) \\
& =\lim _{n \rightarrow \infty}\left(\alpha_{n} \circ \beta_{n}\right)(d)=d \text {. }
\end{aligned}
$$

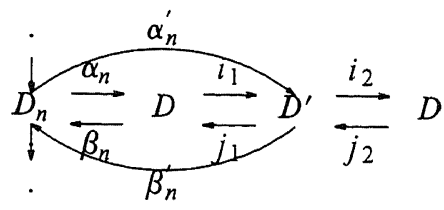

Now we are able to define $D \rightarrow^{\imath} D$ by

$$
\begin{aligned}
\iota & =\iota_{2} \circ \iota_{1} \\
& =<i_{2} \circ i_{1}, j_{1} \circ j_{2}>.
\end{aligned}
$$

It is easy to verify that

$$
\forall n \in \mathbb{N}\left[\iota \circ \gamma_{n}=\gamma_{n}\right] .
$$

By the initiality of $D$ we have that

$$
\iota=\left\langle i d_{D}, i d_{D}>\right.\text {. }
$$

Thus $i_{2} \circ i_{1}=i d_{D}$. This implies $D=D^{\prime}$.

Conclusion:

$$
\lim \alpha_{n} \circ \beta_{n}=i d_{D} .
$$


The initiality lemma will appear to be very useful in the sequel, where we shall construct a cone for an arbitrary converging tower and prove that it is initial.

Definition 3.9 (Direct limit construction)

Let $\left(D_{n}, \iota_{n}\right)_{n}$, with $\iota_{n}=\left\langle i_{n}, j_{n}\right\rangle$, be a converging tower. The direct limit of $\left(D_{n}, \iota_{n}\right)_{n}$ is a cone $\left(D,\left(\gamma_{n}\right)_{n}\right)$, with $\left.\gamma_{n}=<\alpha_{n}, \beta_{n}\right\rangle$, that is defined as follows:

$$
D=\operatorname{def}\left\{\left(x_{n}\right)_{n} \mid \forall n \geqslant 0\left[x_{n} \in D_{n} \wedge j_{n}\left(x_{n+1}\right)=x_{n}\right]\right\}
$$

is equipped with a metric $d: D \times D \rightarrow[0,1]$ such that for all $\left(x_{n}\right)_{n},\left(y_{n}\right)_{n} \in D$ : $d\left(\left(x_{n}\right)_{n},\left(y_{n}\right)_{n}\right)=\sup \left\{d_{D_{n}}\left(x_{n}, y_{n}\right)\right\}$;

$\alpha_{n}: D_{n} \rightarrow D$ is defined by $\alpha_{n}(x)=\left(x_{k}\right)_{k}$, where

$$
x_{k}= \begin{cases}j_{k n}(x) & \text { if } k<n \\ x & \text { if } k=n \\ i_{n k}(x) & \text { if } k>n\end{cases}
$$

$\beta_{n}: D \rightarrow D_{n}$ is defined by $\beta_{n}\left(\left(x_{k}\right)_{k}\right)=x_{n}$.

LEMMA 3.10

Let $(D, d)$ be as defined above. We have:

$$
(D, d) \text { is a complete metric space. }
$$

\section{ProOF}

Let $\left(x_{n}\right)_{n},\left(y_{n}\right)_{n} \in D$. Let $m>n \geqslant 0$, then

$$
\begin{aligned}
d_{D_{n}}\left(x_{n}, y_{n}\right)= & d_{D_{n}}\left(j_{n m}\left(x_{m}\right), j_{n m}\left(y_{m}\right)\right) \\
\leqslant & \text { [ because } j_{n m} \text { is NDI ] } \\
& d_{D_{m}}\left(x_{m}, y_{m}\right) .
\end{aligned}
$$

Thus $\left(d_{D_{n}}\left(x_{n}, y_{n}\right)\right)_{n}$ is an increasing sequence. It is bounded by 1 , thus its supremum exists, and is equal to the limit. It is not difficult to show that $d$ is a metric.

We shall prove the completeness of $D$ with respect to this metric. Let $\left(\bar{x}^{i}\right)_{i}$, with $\bar{x}^{i}=\left(x_{0}^{i}, x_{1}^{i}, x_{2}^{i}, \ldots\right)$ be a Cauchy sequence in $D$. Because for all $k$ and for all $n$ and $m$ :

$$
\begin{aligned}
d_{D_{k}}\left(x_{k}^{n}, x_{k}^{m}\right) & \leqslant \sup _{k \in \mathbb{N}}\left\{d_{D_{k}}\left(x_{k}^{n}, x_{k}^{m}\right)\right\} \\
& =d\left(\bar{x}^{n}, \bar{x}^{m}\right)
\end{aligned}
$$

and $\left(\bar{x}^{i}\right)_{i}$ is a Cauchy sequence, we have, for all $k \in \mathbb{N}$, that $\left(x_{k}^{i}\right)_{i}$ is a Cauchy sequence in $D_{k}$. For every $k$ we set

$$
x_{k}=\lim _{i \rightarrow \infty} x_{k}^{i} .
$$


We have $j_{k}\left(x_{k+1}\right)=x_{k}$, since

$$
\begin{aligned}
j_{k}\left(x_{k+1}\right) & =j_{k}\left(\lim _{i \rightarrow \infty} x_{k+1}^{i}\right) \\
& =\lim _{i \rightarrow \infty} j_{k}\left(x_{k+1}^{i}\right) \\
& =\lim _{i \rightarrow \infty} x_{k}^{i} \\
& =x_{k} .
\end{aligned}
$$

Thus $\left(x_{k}\right)_{k}$ is an element of $D$.

Because the convergence of the sequences $\left(x_{k}^{i}\right)_{i}$ for $k \in \mathbb{N}$ was uniform, we have

$$
\forall \epsilon>0 \exists N \in \mathbb{N} \forall k \in \mathbb{N} \forall n>N\left[d_{D_{k}}\left(x_{k}^{n}, x_{k}\right)<\epsilon\right] .
$$

This fact implies that $\left(x_{k}\right)_{k}$ is the limit of $\left(\bar{x}^{i}\right)_{i}$, since, for $\epsilon>0$,

$$
\begin{aligned}
d\left(\left(x_{k}\right)_{k}, \bar{x}^{n}\right) & =\sup _{k \in \mathbb{N}}\left\{d_{D_{k}}\left(x_{k}, x_{k}^{n}\right)\right\} \\
& \leqslant \epsilon
\end{aligned}
$$

for $n$ bigger than a suitable $N$.

\section{RELATION BETWEEN THE DIRECT LIMIT CONSTRUCTION AND METRIC COMPLETION}

We can look upon the construction of the direct limit for a tower $\left(D_{n}, i_{n}\right)_{n}$ as a generalization of taking the metric completion of the union of a sequence of metric spaces. We define

$$
\begin{aligned}
D_{0}^{\prime} & =\{0\} \times D_{0} \\
D_{n+1}^{\prime} & =\{n+1\} \times\left(D_{n+1} \backslash i_{n}\left(D_{n}\right)\right) \cup D_{n}^{\prime},
\end{aligned}
$$

and take $l_{n}: D_{n} \rightarrow D_{n}^{\prime}$ as follows:

$$
\begin{aligned}
& l_{0}(d)=<0, d>\quad \text { for } d \in D_{0}, \\
& l_{n+1}(d)=\left\{\begin{array}{cl}
l_{n}\left(d^{\prime}\right) & \text { if } d=i_{n}\left(d^{\prime}\right) \in D_{n+1} \\
<n+1, d> & \text { if } d \notin i_{n}\left(D_{n}\right) .
\end{array} \text { with } d^{\prime} \in D_{n}\right.
\end{aligned}
$$

Because each $i_{n}$ is an injection, this construction works, and we see that each $l_{n}$ is a bijection. Therefore, we can use $\left(l_{n}\right)_{n}$ in the obvious way to define a metric $d_{n}$ on each $D_{n}^{\prime}$ and suitable $i_{n}^{\prime}: D_{n}^{\prime} \rightarrow D_{n+1}^{\prime}$ and $j_{n}^{\prime}: D_{n+1}^{\prime} \rightarrow D_{n}^{\prime}$.

Now we have an isomorphic copy of our original tower, which satisfies the condition that each $i_{n}^{\prime}: D_{n}^{\prime} \rightarrow D_{n+1}^{\prime}$ is a subset embedding. From now on we leave out the primes, and just suppose that $i_{n}: D_{n} \rightarrow D_{n+1}$ satisfies this condition.

If we define $U$ as the union of $\left(D_{n}\right)_{n}$, and $d: U \times U \rightarrow[0,1]$ by

$$
d(x, y)=d_{D_{k}}(x, y),
$$

whenever $x \in D_{n}, y \in D_{m}$ and $k \geqslant m, n$, we have that $(U, d)$ is a metric space. Generally, it will not 
be complete. The direct limit of $\left(D_{n}, i_{n}\right)_{n}$ can be regarded as the completion of $(U, d)$ in the following sense.

In $U$ we consider only such sequences $\left(x_{n}\right)_{n}$, for which:

$$
\forall n \in \mathbb{N}\left[x_{n} \in D_{n}\right]
$$

and

$$
\forall n \in \mathbb{N}\left[x_{n}=j_{n}\left(x_{n+1}\right)\right]
$$

It follows that $\left(x_{n}\right)_{n}$ is a Cauchy sequence. For $m>n$ we have

$$
\begin{aligned}
d\left(x_{m}, x_{n}\right) & =d_{D_{m}}\left(x_{m}, i_{n m}\left(x_{n}\right)\right) \\
& =d_{D_{m}}\left(x_{m}, i_{n m} \circ j_{n m}\left(x_{m}\right)\right) \\
& \leqslant d_{D_{m} \rightarrow D_{m}}\left(i d_{D_{m}}, i_{n m} \circ j_{n m}\right) \\
& =\delta\left(\iota_{n m}\right) .
\end{aligned}
$$

This number is small for large $n$ and $m$, because $\left(D_{n}, i_{n}\right)_{n}$ is a converging tower.

For every $\left(x_{n}\right)_{n}$ and $\left(y_{n}\right)_{n}$ in $U$, that both satisfy (1) and (2), we have:

$$
\text { if } \lim _{n \rightarrow \infty} d_{D_{n}}\left(x_{n}, y_{n}\right)=0 \text {, then }\left(x_{n}\right)_{n}=\left(y_{n}\right)_{n},
$$

because of:

$$
\begin{aligned}
d_{D_{n}}\left(x_{n}, y_{n}\right) & =d_{D_{n}}\left(j_{n}\left(x_{n}+1\right), j_{n}\left(y_{n+1}\right)\right) \\
& \leqslant d_{D_{n+1}}\left(x_{n+1}, y_{n+1}\right)
\end{aligned}
$$

(expressing that $\left(d_{D_{n}}\left(x_{n}, y_{n}\right)\right)_{n}$ is a monotonic, non-decreasing sequence with limit 0 , so all its elements are 0 ).

Of course it is not the case that every Cauchy sequence satisfies (1) and (2), but we can find in each class of Cauchy sequences that will have the same limit a representative sequence, which satisfies (1) and (2), and which by the above is unique. Let $\left(x_{n}\right)_{n}$ be an arbitrary Cauchy sequence in $U$. As a representative of the class of Cauchy sequences with the same limit as $\left(x_{n}\right)_{n}$, we take the sequence $\left(y_{n}\right)_{n}$, defined by

$$
y_{n}=\lim _{m \rightarrow \infty} x_{m}^{n},
$$

with

$$
x_{m}^{n}=\left\{\begin{array}{cl}
x_{m} & \text { if } x_{m} \in D_{n} \\
j_{n k}\left(x_{m}\right) & \text { if } x_{m} \notin D_{n}, \text { and } k>n \text { is the least number with } x_{m} \in D_{k}
\end{array}\right.
$$

(Remember that $k>n \Rightarrow D_{k} \supset D_{n}$ ). It is not very difficult to show, that we have indeed:

$$
\lim _{n \rightarrow \infty} d_{D_{n}}\left(x_{n}, y_{n}\right)=0,
$$


and that $\left(y_{n}\right)_{n}$ satisfies (1) and (2). Finally we remark that the direct limit $D$ of $\left(D_{n}, \iota_{n}\right)_{n}$ consists of exactly those sequences in $U$, that satisfy (1) and (2), and thus can be viewed as the metric completion of $(U, d)$.

Remember from theorem 2.9 that the metric completion $\bar{M}$ of a metric space $M$ is the smallest complete metric space, into which $M$ can be isometrically embedded, in the following sense: $\bar{M}$ can be isometrically embedded into every other complete metric space with that property.

For the direct limit of a converging tower, we have a similar initiality property:

\section{LEMMA 3.11}

The direct limit of a converging tower (as defined in definition 3.9) is an initial cone for that tower.

\section{Proof}

Let $\left(D_{n}, \iota_{n}\right)_{n}$ and $\left(D,\left(\gamma_{n}\right)_{n}\right)$ be as defined in definition 3.9. According to the initiality lemma (3.9), it suffices to prove

$$
\lim _{n \rightarrow \infty} \alpha_{n} \circ \beta_{n}=i d_{D},
$$

which is equivalent to

$$
\forall \epsilon>0 \exists N \in \mathbb{N} \forall n>N\left[d\left(\alpha_{n} \circ \beta_{n}, i d_{D}\right)<\epsilon\right]
$$

Let $\epsilon>0$. Because $\left(D_{n}, \iota_{n}\right)_{n}$ is a converging tower, we can choose $N \in \mathbb{N}$ such that

$$
\forall m>n \geqslant N\left[d\left(i_{n m} \circ j_{n m}, i d_{D_{m}}\right)<\epsilon\right] .
$$

Let $n>N$. Let $\left(x_{m}\right)_{m} \in D$, we define

$$
\left(y_{m}\right)_{m}=\alpha_{n} \circ \beta_{n}\left(\left(x_{m}\right)_{m}\right) .
$$

For every $m>n$ we have

$$
\begin{aligned}
d_{D_{m}}\left(y_{m}, x_{m}\right) & =d_{D_{m}}\left(i_{n m}\left(x_{n}\right), x_{m}\right) \\
& =d_{D_{m}}\left(i_{n m} \circ j_{n m}\left(x_{m}\right), x_{m}\right) \\
& \leqslant d\left(i_{n m} \circ j_{n m}, i d_{D_{m}}\right) \\
& <\epsilon .
\end{aligned}
$$

Therefore

$$
d_{D}\left(\left(y_{m}\right)_{m},\left(x_{m}\right)_{m}\right)=\sup \left\{d_{D_{m}}\left(y_{m}, x_{m}\right)\right\} \leqslant \epsilon .
$$

Because $\left(x_{n}\right)_{n} \in D$ was arbitrary, we have

$$
d\left(\alpha_{n} \circ \beta_{n}, i d_{D}\right)<\epsilon
$$


for all $n>N$.

\subsection{A fixed-point theorem}

As a category-theoretic equivalent of a contracting function on a metric space, we have the following notion of a contracting functor on $\mathrm{C}$.

DeFINITION 3.12 (Contracting functor)

We call a functor $F: \varrho \rightarrow \circlearrowright$ contracting whenever the following holds: there exists an $\epsilon$, with $0 \leqslant \epsilon<1$, such that for all $D \rightarrow^{\imath} E \in \mathcal{C}$ we have:

$$
\delta(F \iota) \leqslant \epsilon \cdot \delta(\iota)
$$

A contracting function on a complete metric space is continuous, so it preserves Cauchy sequences and their limits. Similarly, a contracting functor preserves converging towers and their initial cones:

LEMMA 3.13

Let $F: \circlearrowright \rightarrow \circlearrowright$ be a contracting functor, let $\left(D_{n}, l_{n}\right)_{n}$ be a converging tower with an initial cone $\left(D,\left(\gamma_{n}\right)_{n}\right)$. Then $\left(F D_{n}, F l_{n}\right)_{n}$ is again a converging tower with $\left(F D,\left(F \gamma_{n}\right)_{n}\right)$ as an initial cone.

The proof, which may use the initiality lemma, is left to the reader.

Theorem 3.14 (Fixed-point theorem)

Let $F$ be a contracting functor $F: \hookrightarrow \rightarrow e$ and let $D_{0} \rightarrow{ }^{l_{0}} F D_{0} \in C$. Let the tower $\left(D_{n}, \iota_{n}\right)_{n}$ be defined by $D_{n+1}=F D_{n}$ and $\iota_{n+1}=F \iota_{n}$ for all $n \geqslant 0$. This tower is converging, so it has a direct limit $\left(D,\left(\gamma_{n}\right)_{n}\right)$. We have: $D \cong F D$.

\section{Proof}

First we observe that $\left(D_{n}, \iota_{n}\right)_{n}$ can be proved to be a converging tower in the same way as in example 3.4. Because $F$ preserves converging towers and their initial cones, $\left(F D_{n}, F \iota_{n}\right)_{n}$ is again a converging tower with $\left(F D,\left(F \gamma_{n}\right)_{n}\right)$ as an initial cone. We have that

$$
\left(F D_{n}, F \iota_{n}\right)_{n}=\left(D_{n+1}, \iota_{n+1}\right)_{n},
$$

so $\left(F D_{n}, F \iota_{n}\right)_{n}$ has the same direct limit (up to isometry) as $\left(D_{n}, \iota_{n}\right)_{n}$. This implies that $\left(D,\left(\gamma_{n}\right)_{n}\right)$ and $\left(F D,\left(F \gamma_{n}\right)_{n}\right)$ are both initial cones of $\left(D_{n+1}, \iota_{n+1}\right)_{n}$. It follows from the definition of an initial cone that $D$ and $F D$ are isometric. 


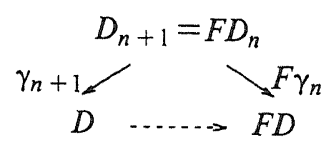

\section{REMARK}

It is always possible to find an arrow $D_{0} \rightarrow{ }^{6} F D_{0} \in \mathcal{C}$ : Take $D_{0}=\left\{p_{0}\right\}$; because $F D_{0}$ is nonempty we can choose an arbitrary $p_{1} \in F D_{0}$, and put $\iota_{0}=<i_{0}, j_{0}>$ with $i\left(p_{0}\right)=p_{1}$ and $j(x)=p_{0}$, for $x \in F D_{0}$.

\section{UNIQUENESS OF FIXED POINTS}

We know that a contracting function $f: M \rightarrow M$, on a complete metric space $M$, has a unique fixed point. We would like to prove a similar property for contracting functors on $C$.

Let us consider a contracting functor $F$ on the category of complete metric spaces $C$. By theorem 3.14 we know that $F$ has a fixed point, that is there exists $D \in \mathcal{C}$ and an isometry $\kappa$ such that

$$
\stackrel{\kappa^{\prime}}{\cong} F D \text {. }
$$

Suppose we have another fixed point $D^{\prime}$ with an isometry $\lambda$, such that

$$
\stackrel{\lambda}{\stackrel{\lambda}{\rightrightarrows} F D^{\prime}}
$$

We know by the construction of $D$ that it is the direct limit of the converging tower $\left(D_{n}, l_{n}\right)_{n}$, where $D_{0} \rightarrow{ }^{\natural} F D_{0} \in \mathcal{C}$ is a given embedding and $D_{n+1}=F D_{n}, \iota_{n+1}=F \iota_{n}$.

If we have that $D^{\prime}$ is also (the endpoint of) a cone for that tower, the initiality of $D$ implies that there exists an isometric embedding $D \rightarrow^{\iota} D^{\prime} \in \mathcal{C}$. If we moreover can demonstrate that this $\iota$ is an isometry, then we can conclude that the functor $F$ has a unique fixed point, which would be quite satisfactory.

A proof for $\iota$ being an isometry might look like:

$$
\begin{aligned}
\delta(\iota)= & (?) \delta\left(F_{\iota}\right) \\
& \leqslant \epsilon \cdot \delta(\iota),
\end{aligned}
$$

implying (once the question-mark has been eliminated) that $\delta(\iota)=0$, thus $\iota$ is an isometry.

It turns out that we can guarantee that the second fixed point $D^{\prime}$ is also a cone for the converging tower $\left(D_{n}, \iota_{n}\right)_{n}$ in one of two ways. Firstly, we can restrict our functor $F$ to the base-point 
category of complete metric spaces (to be defined in a moment). Secondly, we can require $F$ to be contracting in yet another sense, to be called hom-contracting below.

We shall proceed in both directions, first exploring the unicity of fixed points of contracting functors on the base-point category, then focusing on functors on $e$ that are contracting and homcontracting.

In both cases it appears to be possible to prove the equality marked by (?) above. Unfortunately (for good mathematicians, who are said to be lazy), this takes some serious effort, to which the proof of the following theorem bears witness.

First we give the definition of the base-point category:

Definition 4.1 (Base-point category of complete metric spaces)

Let $\bigodot^{*}$ denote the base-point category of complete metric spaces, which has triples

$$
<M, d, m>
$$

for its objects. Here $(M, d)$ is a complete metric space and $m$ is an arbitrary element of $M$, called

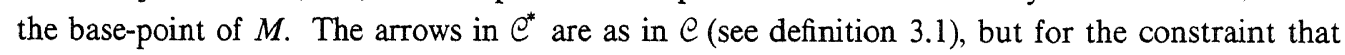
they map base-points onto base-points, i.e. for $\langle M, d, m>\rightarrow<i, j\rangle\left\langle M^{\prime}, d^{\prime}, m^{\prime}\right\rangle \in e^{*}$ we also require that $i(m)=m^{\prime}$, and $j\left(m^{\prime}\right)=m$.

\section{REMARK}

The definitions of cone, functor etcetera can be adapted straightforwardly. Moreover, lemmas 3.8, 3.11, 3.13 and theorem 3.14 still hold.

THEOREM 4.2 (Uniqueness of fixed points)

Let $F$ be a contracting functor $F: e^{*} \rightarrow e^{*}$. Then $F$ has a unique fixed point up to isometry, that is to say: there exists a $D \in C^{*}$ such that

(1) $F D \cong D$, and

(2) $\forall D^{\prime} \in e^{*}\left[F D^{\prime} \cong D^{\prime} \Rightarrow D \cong D^{\prime}\right]$.

\section{Proof}

We define a converging tower $\left(D_{n}, \iota_{n}\right)_{n}$ by

$$
\begin{aligned}
& D_{0}=<\left\{p_{0}\right\}, d_{\left\{p_{0}\right\}}, p_{0}>, \\
& D_{n+1}=F D_{n} \text { for all } n \geqslant 0, \\
& \iota_{0}: D_{0} \rightarrow D_{1}, \text { trivial, } \\
& \iota_{n+1}=F \iota_{n} \text { for all } n \geqslant 0 .
\end{aligned}
$$

Let $\left(D,\left(\gamma_{n}\right)_{n}\right)$ be the direct limit of this tower. As in theorem 3.14, we have that both $\left(D,\left(\gamma_{n}\right)_{n}\right)$ 
and $\left(F D,\left(F \gamma_{n}\right)_{n}\right)$ are initial cones of $\left(D_{n}, \iota_{n}\right)_{n}$. The initiality of $\left(D_{n},\left(\gamma_{n}\right)_{n}\right)$ implies the existence of a unique arrow $D \rightarrow{ }^{\kappa} F D$, such that for $n \geqslant 0$,

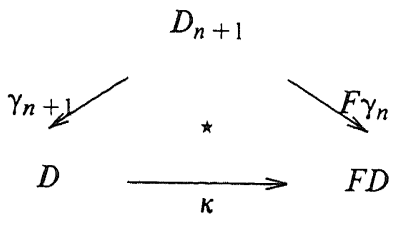

\section{Figure 1}

Because also $\left(F D,\left(F \gamma_{n}\right)\right)_{n}$ is initial, we know that $\kappa$ must be isometric.

Now let $D^{\prime} \in e^{*}$ be another fixed point of $F$, say $\stackrel{\lambda}{D^{\prime}} \underset{\cong}{\dddot{\lambda}} F D^{\prime}$ for an isometry $\lambda$. We define $\left(\tilde{\gamma}_{n}\right)_{n}$ such that $\left(D^{\prime},\left(\tilde{\gamma}_{n}\right)_{n}\right)$ is a cone for $\left(D_{n}, \iota_{n}\right)_{n}$ :

$\tilde{\gamma}_{0}: D_{0} \rightarrow D^{\prime}$ is the unique arrow, which maps base-point to base-point,

$\tilde{\gamma}_{n+1}=\lambda^{-1} \circ F \tilde{\gamma}_{n}$.

We have that $\left(D^{\prime},\left(\tilde{\gamma}_{n}\right)_{n}\right)$ is indeed a cone for $\left(D_{n}, \iota_{n}\right)_{n}$ because of the commutativity of the following diagram, for all $n \in \mathbb{N}$ :

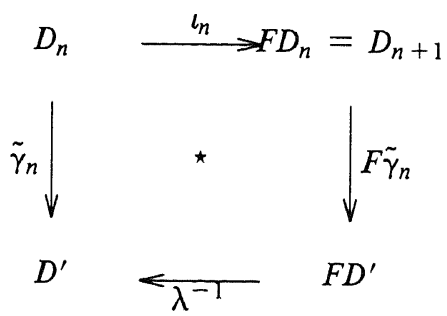

We prove it by induction on $n$ :

(0) Because the arrows in $C^{\star}$ map base-points onto base-points, we have that $\left(\lambda^{-1} \circ F \tilde{\gamma}_{0}{ }^{\circ} \varphi_{0}\right)_{1}\left(p_{0}\right)$ and $\left(\tilde{\gamma}_{0}\right)_{1}\left(p_{0}\right)$ are both equal to the base-point of $D^{\prime}$, and for any $x \in D^{\prime}$, that $\left(\lambda^{-1} \circ F \tilde{\gamma}_{0} \circ \iota_{0}\right)_{2}(x)=\left(\tilde{\gamma}_{0}\right)_{2}(x)=p_{0}$.

Note that this is the only place, where we make use of the base-point structure of $e^{\star}$.

$(n+1)$ Suppose that we have $\lambda^{-1} \circ F \tilde{\gamma}_{n} \circ \iota_{n}=\tilde{\gamma}_{n}$. Then

$$
\begin{aligned}
\lambda^{-1} \circ F \tilde{\gamma}_{n+1} \circ \iota_{n+1} & =\lambda^{-1} \circ F\left(\tilde{\gamma}_{n+1} \circ \iota_{n}\right) \\
& =\lambda^{-1} \circ F\left(\lambda^{-1} \circ F \tilde{\gamma}_{n} \circ \iota_{n}\right)
\end{aligned}
$$




$$
\begin{aligned}
& =\lambda^{-1} \circ F \tilde{\gamma}_{n} \\
& =\tilde{\gamma}_{n+1} .
\end{aligned}
$$

Again by the initiality of $\left(D,\left(\gamma_{n}\right)_{n}\right)$ there is a unique arrow $D \rightarrow^{\prime} D^{\prime}$ such that, for all $n \in \mathbb{N}$ :

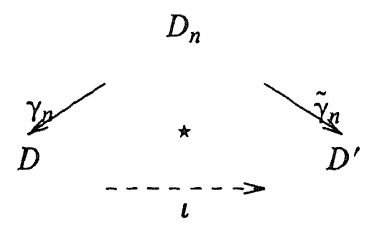

Figure 2

As indicated above, we now set out to prove that $\iota$ is an isometry. When we apply $F$ to figure 2, we get

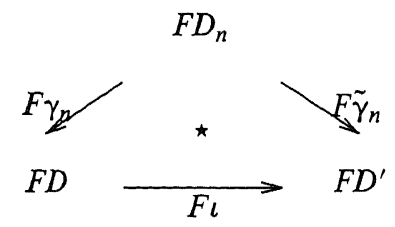

which leads to:

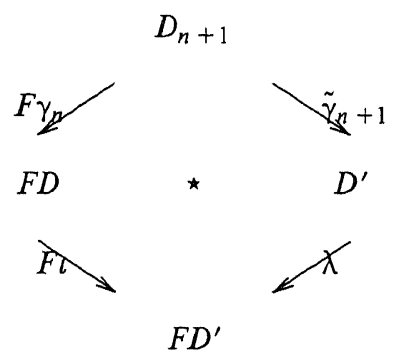

(because $\tilde{\gamma}_{n+1}=\lambda^{-1} \circ F \tilde{\gamma}_{n}$, so $F \tilde{\gamma}_{n}=\lambda \circ \gamma_{n+1}$ ), or, replacing $\lambda$ by $\lambda^{-1}$ and reversing the corresponding arrow: 


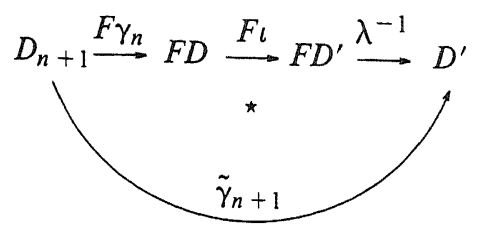

Substituting $\kappa \circ \gamma_{n+1}$ for $F \gamma_{n}$ (figure 1) yields:

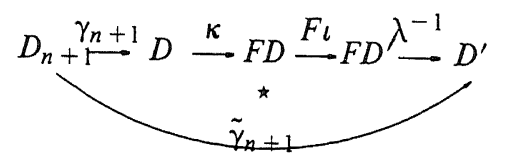

or: $\left(\lambda^{-1} \circ F \iota \circ \kappa\right) \circ \gamma_{n+1}=\tilde{\gamma}_{n+1}$ (this equality also holds for $\gamma_{0}$ and $\tilde{\gamma}_{0}$ ). But according to figure 2 , $\iota$ is the only arrow with: $\forall n \in \mathbb{N}\left[\iota \circ \gamma_{n}=\tilde{\gamma}_{n}\right]$. Thus

$$
\iota=\lambda^{-1} \circ F \iota \circ \kappa,
$$

or, in other words:

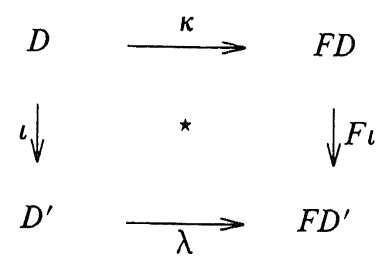

This commutativity, together with the fact that $\kappa$ and $\lambda$ are isometries implies:

$$
\delta(\iota)=\delta(F \iota) \text {. }
$$

(For the definition of $\delta$ see definition 3.2.)

Now the proof can be concluded, following the train of thought indicated above:

$$
\begin{aligned}
\delta(\iota) & =\delta(F \iota) \\
& \leqslant \epsilon \cdot \delta(\iota),
\end{aligned}
$$

for some $0 \leqslant \epsilon<1$, since $F$ is a contraction. This implies

$$
\delta(\iota)=0,
$$

so (if $\iota=<i, j>$ )

$$
i \circ j=i d_{D^{\prime}} .
$$

At last we can draw the desired conclusion: 


$$
\stackrel{\imath}{\cong} D^{\prime}
$$

Now we return again to our original category $e$ of complete metric spaces and provide for, as promised above, another criterion for functors on $\mathcal{C}$, that, together with contractivity, will appear to be sufficient to ensure uniqueness of their fixed points.

DeFINITION 4.3 (Hom-contractivity)

We call a functor $F: \circlearrowright \rightarrow e$ hom-contracting, whenever

$$
\forall P \in \bigodot \forall Q \in \bigodot \exists \epsilon<1\left[F_{P, Q}:\left(P \rightarrow{ }^{\complement} Q\right) \rightarrow \rightarrow^{\epsilon}\left(F P \rightarrow{ }^{\complement} F Q\right)\right]
$$

where

$$
P \rightarrow{ }^{\complement} Q=\{\iota|\iota: P \rightarrow Q| \iota \text { is an arrow in } C\}, \quad F_{P, Q}(\iota)=F \iota .
$$

\section{REMARKS}

Because arrows in $e$ are pairs, we have on $P \rightarrow{ }^{e} Q$ the standard metric for the Cartesian product. So let $\iota_{1}, \iota_{2}: P \rightarrow Q, \iota_{1}=<i_{1}, j_{1}>$ and $\left.\iota_{2}=<i_{2}, j_{2}\right\rangle$. Then their distance is defined by

$$
d\left(\iota_{1}, \iota_{2}\right)=\max \left\{d_{P \rightarrow Q}\left(i_{1}, i_{2}\right), d_{Q \rightarrow P}\left(j_{1}, j_{2}\right)\right\} .
$$

It is not the case that every hom-contracting functor is also contracting, which follows from the following example.

Let $A=\{0\}$ and $B=\{1,2\}$ be discrete metric spaces. We define a functor $F: \circlearrowright \rightarrow \bigodot$ as follows. For every complete metric space $P \in \mathcal{C}$ let

$$
F P= \begin{cases}A & \text { if } P \text { contains exactly } 1 \text { element } \\ B & \text { otherwise. }\end{cases}
$$

For $\iota: P \rightarrow Q$ we define $F \iota$ :

$$
F \iota= \begin{cases}1_{A} & \text { if } F P=F Q=A \\ l_{B} & \text { if } F P=F Q=B \\ \wp & \text { if } F P=A \text { and } F Q=B,\end{cases}
$$

where $\iota_{0}=\left\langle i_{0}, j_{0}\right\rangle$, with $i_{0}: 0 \mapsto 1, j_{0}: 1,2 \mapsto 0$. Note that there is no $\iota: P \rightarrow Q$ if $F P=B$ and $F Q=A$. It is not difficult to verify that $F$ is a functor, which is hom-contracting. The following argument shows that it is not contracting. Let $C=\{3,4\}$ with $d(3,4)=\frac{1}{2}$, and let $\kappa: A \rightarrow C$, with $\kappa=\left\langle k, l>\right.$ be defined by $k: 0 \mapsto 3$ and $l: 3,4 \mapsto 0$. Then we have $\delta(\kappa)=\frac{1}{2}$, but $F \kappa: F A \rightarrow F C$ is $\iota_{0}: A \rightarrow B$ (as defined above), for which $\delta\left(\iota_{0}\right)=1$. 


\section{THEOREM 4.4}

Let $F$ be a contracting and hom-contracting functor $F: \circlearrowright \rightarrow \circlearrowright$. Then $F$ has a unique fixed point up to isometry, that is to say: there exists a $D \in \mathcal{C}$ such that
(1) $F D \cong D$ and
(2) $\forall D^{\prime} \in \mathcal{C}^{*}\left[F D^{\prime} \cong D^{\prime} \Rightarrow D \cong D^{\prime}\right]$.

PROOF

The proof of this theorem differs from that of theorem 4.2 only in the definition of $\tilde{\gamma}_{0}$. There we could take for $\tilde{\gamma}_{0}$ the trivial embedding of $D_{0}$ into $D^{\prime}$, mapping $p_{0}$ onto the base-point of $D^{\prime}$. Here we have no base-points. But we can use the fact that $F$ is hom-contracting by taking for $\tilde{\gamma}_{0}$ the unique fixed point of the function $G:\left(D_{0} \rightarrow{ }^{e} D^{\prime}\right) \rightarrow\left(D_{0} \rightarrow{ }^{e} D^{\prime}\right)$, that we define by: $G(\tilde{\gamma})=\lambda^{-1} \circ F \tilde{\gamma}^{\circ} \iota_{0}$, for $\tilde{\gamma} \in\left(D_{0} \rightarrow^{e} D^{\prime}\right)$. (Note that $G$ is contracting because $F$ is hom-contracting.) It follows that $\tilde{\gamma}_{0}$, thus defined, satisfies $\lambda^{-1} \circ F \tilde{\gamma}_{0}{ }^{\circ} \varphi_{0}=\tilde{\gamma}_{0}$, which serves our purposes.

\section{A CLASS OF DOMAIN EQUATIONS WITH UNIQUE SOLUTIONS}

In this section we present a class of domain equations over the category $e$ that have unique solutions. For this purpose we first define a set Func of functors on $e$ and formulate a condition for its elements that implies contractivity and hom-contractivity. It then follows that every domain equation over $C$ induced by a functor that satisfies this condition, has a unique solution.

\section{DeFINITION 5.1 (Functors)}

The set Func, with typical elements $F$, is defined by:

$$
F::=F_{M}\left|i d^{\epsilon}\right| F_{1} \rightarrow F_{2}\left|F_{1} \rightarrow^{1} F_{2}\right| F_{1} \bar{\cup} F_{2}\left|F_{1} \times F_{2}\right| \mathscr{P}_{c l}(F) \mid F_{1} \circ F_{2}
$$

where $M$ is an arbitrary complete metric space and $\epsilon>0$. Every $F \in$ Func is to be interpreted as a functor

$$
F: \circlearrowright \rightarrow \bigodot
$$

as follows. Let $\left(P, d_{P}\right),\left(Q, d_{Q}\right) \in e$ be complete metric spaces. Let $P \rightarrow^{\iota} Q \in \varrho$, with $\iota=<i, j>$. For the definition of each $F \in F u n c$ we have to specify:

(1) the image of $P$ under $F: F P$,

(2) the image of $d$ under $F: F d$,

(3) the image of $\iota$ under $F: F \iota(=<F i, F j>)$. 
(a) $F=F_{M}$ :
(1) $F P=M$,
(2) $F d=d_{M}$ (the metric of $\left.M\right)$,
(3) $F \iota=\left\langle i d_{M}, i d_{M}>\right.$.

We sometimes use just a set $A$ instead of a metric space $M$. In this case we provide $A$ with the discrete metric (definition 2.1).

(b) $F=i d^{6}$ :
(1) $F P=P$,
(2) $F d=\epsilon \cdot d(F d(x, y)=\epsilon \cdot d(x, y)$, for $x, y \in P)$,
(3) $F \iota=\iota$

Next we define functors that are composed. Let $F_{1}, F_{2} \in$ Func, such that
(1) $F_{1} P=P_{1}, F_{2} P=P_{2}, F_{1} Q=Q_{1}, F_{2} Q=Q_{2}$,
(2) $F_{1} d=d_{1}, F_{2} d=d_{2}$,
(3) $F_{1} \iota=\left\langle i_{1}, j_{1}\right\rangle, F_{2} \iota=\left\langle i_{2}, j_{2}\right\rangle$.

(c) $F=F_{1} \rightarrow F_{2}$ :

(1) $F P=P_{1} \rightarrow P_{2}$,

(2) $F d=d_{F}$ (see definition 2.6(a)),

(3) $F_{\iota}=\left\langle\lambda f \cdot\left(i_{2} \circ f^{\circ} j_{1}\right), \lambda g \cdot\left(j_{2} \circ g \circ i_{1}\right)>\right.$.

( $F=F_{1} \rightarrow^{1} F_{2}$ is defined similarly.)

(d) $F=F_{1} \cup F_{2}$ :
(1) $F P=P_{1} \bar{\cup} P_{2}$
(2) $F d=d_{U}$ (see definition 2.6(b)),
(3) $F_{\iota}=<\lambda p \cdot$ if $p \in\{0\} \times P_{1}$ then $i_{1}\left((p)_{2}\right)$ else $i_{2}\left((p)_{2}\right)$ fi, $\lambda q \cdot$ if $q \in\{0\} \times Q_{1}$ then $j_{1}\left((q)_{2}\right)$ else $j_{2}\left((q)_{2}\right)$ fi $>$.

(e) $F=F_{1} \times F_{2}$ :
(1) $F P=P_{1} \times P_{2}$,
(2) $F d=d_{P}($ see definition $2.6(\mathrm{c}))$,
(3) $F \iota=\left\langle\lambda\left\langle p_{1}, p_{2}\right\rangle \cdot\left\langle i_{1}\left(p_{1}\right), i_{2}\left(p_{2}\right)\right\rangle, \lambda<q_{1}, q_{2}\right\rangle \cdot\left\langle j_{1}\left(q_{1}\right), j_{2}\left(q_{2}\right)\right\rangle>$. 
(f) $F=\rho_{c l}\left(F_{1}\right)$ :

(1) $F P=\mathscr{P}_{c l}\left(P_{1}\right)$,

(2) $F d=d_{H}$ (see definition 2.6(d)),

(3) $F_{\iota}=<\lambda X \cdot\left\{i_{1}(x) \mid x \in X\right\}, \lambda Y \cdot$ closure $\left\{j_{1}(y) \mid y \in Y\right\}>$.

(g) $F=F_{1} \circ F_{2}$ : the usual composition of functors on $C$.

\section{REMARK}

The set Func contains elements of various form. We give an example. Let $F_{1}, F_{2} \in$ Func. The following functor is an element of the set Func, as can be deduced from its definition.

$$
F_{1} \rightarrow^{A} F_{2}={ }^{d e f} i d^{A} \circ\left(F_{1} \rightarrow^{1}\left(i d^{\frac{1}{A}} \circ F_{2}\right)\right) \text {, for } A>0 \text {. }
$$

\section{LEMMA 5.2}

For all $F \in$ Func we have: $F$ is a well defined functor on $e$

\section{Proof}

We treat only one case by way of example, being (lazy and) confident that it shows the reader how to proceed in the other cases.

Let $F=F_{1} \rightarrow^{1} F_{2}$, and suppose $F_{1}$ and $F_{2}$ are well defined. Let $\left(P, d_{P}\right),\left(Q, d_{Q}\right)$ and $P \rightarrow{ }^{l} Q \in \mathcal{C}$, with $\iota=\langle i, j\rangle$; furthermore, let for $k=1,2$ :

$$
\begin{aligned}
& F_{k} P=P_{k}, \quad F_{k} Q=Q_{k}, \\
& F_{k} d_{P}=d_{P_{k}}, \quad F_{k} d_{Q}=d_{Q_{k}}, \\
& F_{k} \iota=<i_{k}, j_{k}>.
\end{aligned}
$$

The functor $F$ is defined by

(1) $F P=P_{1} \rightarrow^{1} P_{2}$,

(2) $F d_{P}=d_{F}$,

(3) $F i=\langle F i, F j\rangle=\left\langle\lambda f \cdot\left(i_{2} \circ f^{\circ} j_{1}\right), \lambda g \cdot\left(j_{2} \circ g \circ i_{1}\right)\right\rangle$.

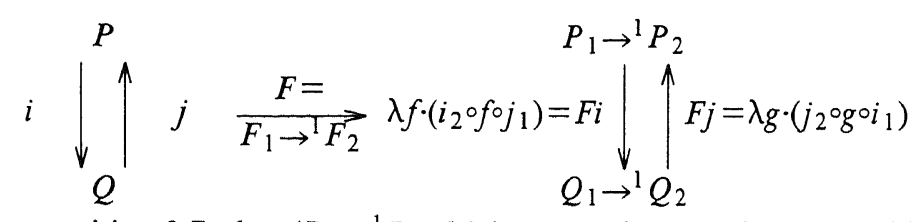

It follows from proposition 2.7 , that $\left(P_{1} \rightarrow{ }^{1} P_{2}, d_{F}\right)$ is a complete metric space, which leaves us to prove: 
(a) Fi is isometric,

(b) $F j$ is NDI and

(c) $F j \circ F i=i d_{F P}$.

Part (a): Let $f_{1}, f_{2} \in P_{1} \rightarrow{ }^{1} P_{2}$. We want to show

$$
d_{F P}\left(f_{1}, f_{2}\right)=d_{F Q}\left(F i\left(f_{1}\right), F i\left(f_{2}\right)\right) .
$$

We have

$$
\begin{aligned}
\sup _{q \in Q_{1}}\left\{d_{Q_{2}}\left(i_{2} \circ f_{1} \circ j_{1}(q), i_{2} \circ f_{2} \circ j_{1}(q)\right)\right\}= & {\left[\text { because } i_{2}\right. \text { is isometric] }} \\
& \sup _{q \in Q_{1}}\left\{d_{P_{2}}\left(f_{1} \circ j_{1}(q), f_{2} \circ j_{1}(q)\right)\right\} \\
= & {\left[\text { because } j_{1} \text { is surjective }\right] } \\
& \sup _{p \in P_{1}}\left\{d_{P_{2}}\left(f_{1}(p), f_{2}(p)\right)\right\} \\
= & d_{P_{1} \rightarrow P_{2}}\left(f_{1}, f_{2}\right) .
\end{aligned}
$$

Part (b): Let $g_{1}, g_{2} \in Q_{1} \rightarrow^{1} Q_{2}$. We want to show:

$$
d_{F P}\left(F j\left(g_{1}\right), F j\left(g_{2}\right)\right) \leqslant d_{F Q}\left(g_{1}, g_{2}\right) .
$$

Let $p \in P_{1}$; we have:

$$
\begin{aligned}
d_{P_{2}}\left(F j\left(g_{1}\right)(p), F j\left(g_{2}\right)(p)\right)= & d_{P_{2}}\left(j_{2} \circ g_{1} \circ i_{1}(p), j_{2} \circ g_{2} \circ i_{1}(p)\right) \\
\leqslant & {\left[j_{2}\right. \text { is NDI] }} \\
& d_{Q_{2}}\left(g_{1} \circ i_{1}(p), g_{2} \circ i_{1}(p)\right) \\
\leqslant & d_{F Q}\left(g_{1}, g_{2}\right) .
\end{aligned}
$$

Part (c): Let $f \in P_{1} \rightarrow{ }^{1} P_{2}$. We have

$$
\begin{aligned}
F j \circ F i(f) & =j_{2} \circ i_{2} \circ f^{\circ} j_{1} \circ i_{1} \\
& =f .
\end{aligned}
$$

Definition 5.3 (Contraction coefficient)

For each $F \in$ Func we define its so-called contraction coefficient (notation: $c(F)$, with $c(F) \in[0, \infty])$, using induction on the complexity of the structure of $F$.

(a) If $F=F_{M}$, then $c(F)=0$.

(b) If $F=i d^{\epsilon}$, then $c(F)=\epsilon$. 
Let $F_{1}, F_{2} \in F u n c$, with coefficients $c\left(F_{1}\right)$ and $c\left(F_{2}\right)$. Then we set:

(c) If $F=F_{1} \rightarrow F_{2}$, then $c(F)=\max \left\{\infty \cdot c\left(F_{1}\right), c\left(F_{2}\right)\right\}$.

(d) If $F=F_{1} \rightarrow^{1} F_{2}$, then $c(F)=c\left(F_{1}\right)+c\left(F_{2}\right)$.

(If we would restrict ourselves to ultra-metric spaces, we could write $\max \left\{c\left(F_{1}\right), c\left(F_{2}\right)\right\}$ here.)

(e) If $F=F_{1} \bar{\cup} F_{2}$, then $c(F)=\max \left\{c\left(F_{1}\right), c\left(F_{2}\right)\right\}$.

(f) If $F=F_{1} \times F_{2}$, then $c(F)=\max \left\{c\left(F_{1}\right), c\left(F_{2}\right)\right\}$.

(g) If $F=\rho_{c l}\left(F_{1}\right)$, then $c(F)=c\left(F_{1}\right)$.

(h) If $F=F_{1} \circ F_{2}$, then $c(F)=c\left(F_{1}\right) \cdot c\left(F_{2}\right)$.

(With $\infty$ we compute as follows: $\infty \cdot 0=0 \cdot \infty=0, \infty \cdot c=c \cdot \infty=\infty$, if $c>0$.)

\section{THEOREM 5.4}

For every functor $F \in$ Func we have

(1) $\forall P \rightarrow^{\iota} Q \in \circlearrowright[\delta(F \iota) \leqslant c(F) \cdot \delta(\iota)]$,

(2) $\forall P, Q \in \mathcal{C}\left[F_{P, Q}:\left(P \rightarrow^{\complement} Q\right) \rightarrow^{c(F)}\left(F P \rightarrow^{e} F Q\right)\right]$.

\section{ProOF}

Let $P, Q \in \mathcal{C}, \iota, \iota^{\prime} \in P \rightarrow{ }^{\complement} Q$, with $\left.\iota=\langle i, j\rangle, \iota^{\prime}=<i^{\prime}, j^{\prime}\right\rangle$.

Case (a) $F=F_{M}$ :

Part (al)

$$
\begin{aligned}
\delta(F \iota) & =d_{F Q \rightarrow F Q}\left(F i \circ F j, i d_{M}\right) \\
& =d_{F Q \rightarrow F Q}\left(i d_{M} \circ i d_{M}, i d_{M}\right) \\
& =0=c(F) \cdot \delta(\iota) .
\end{aligned}
$$

part (a2)

$$
d_{F P \rightarrow} e_{F Q}\left(F \iota, F \iota^{\prime}\right)=d_{M \rightarrow} e_{M}(i d, i d)=0=c(F) \cdot d_{p \rightarrow}{ }^{e} Q\left(\iota, \iota^{\prime}\right) .
$$

Case (b) $F=i d^{\epsilon}$ :

part (b1)

$$
\begin{aligned}
\delta(F \iota) & =d_{F Q \rightarrow F Q}\left(F i \circ F j, i d_{F Q}\right) \\
& =\sup _{q \in Q}\left\{d_{F Q}(i \circ j(q), q)\right\} \\
& =\sup _{q \in Q}\left\{\epsilon \cdot d_{Q}(i \circ j(q), q)\right\} \\
& =\epsilon \cdot \delta(\iota)
\end{aligned}
$$




$$
=c(F) \cdot \delta(\iota)
$$

Part (b2)

$$
\begin{aligned}
& d_{F P \rightarrow{ }^{e}} F Q\left(F \iota, \iota^{\prime}\right)=\epsilon \cdot d_{P \rightarrow{ }^{e} Q}\left(\iota, \iota^{\prime}\right) \\
& =c(F) \cdot d_{P \rightarrow} e_{Q}\left(\iota, \iota^{\prime}\right) .
\end{aligned}
$$

Now let $F_{1}, F_{2} \in$ Func and suppose the theorem holds for these functors. For $k=1,2$ we use the following notation:

$$
\begin{aligned}
& F_{k} \iota=\iota_{k}, F_{k} \iota^{\prime}=i_{k}^{\prime}, F_{k} P=P_{k}, F_{k} Q=Q_{k}, \\
& F_{k} i=i_{k}, F_{k} i^{\prime}=i_{k}^{\prime}, \\
& F_{k} j=j_{k}, F_{k} j^{\prime}=j_{k}^{\prime},
\end{aligned}
$$

We only treat the cases that $F=F_{1} \rightarrow^{1} F_{2}$ and $F=F_{1} \times F_{2}$.

Case (d) $F=F_{1} \rightarrow^{1} F_{2}$ :

Part (dl)

$$
\begin{aligned}
\delta(F \iota) & =d_{F Q \rightarrow F Q}\left(F i \circ F j, i d_{F Q}\right) \\
& =\sup _{g \in F Q}\left\{d_{F Q}\left(i_{2} \circ j_{2} \circ g \circ i_{1} \circ j_{1}, g\right)\right\} .
\end{aligned}
$$

Let $g \in F Q=Q_{1} \rightarrow^{1} Q_{2}$. For $q_{1} \in Q_{1}$ we have

$$
\begin{aligned}
d_{Q_{2}}\left(i_{2} \circ j_{2} \circ g \circ i_{1} \circ j_{1}\left(q_{1}\right), g\left(q_{1}\right)\right) \leqslant & d_{Q_{2}}\left(i_{2} \circ j_{2} \circ g \circ i_{1} \circ j_{1}\left(q_{1}\right), g \circ i_{1} \circ j_{1}\left(q_{1}\right)\right)+ \\
& d_{Q_{2}}\left(g \circ i_{1} \circ j_{1}\left(q_{1}\right), g\left(q_{1}\right)\right) .
\end{aligned}
$$

(This "+" could be replaced by "max" in the case of ultra-metric spaces.)

For the first term we have

$$
\begin{gathered}
d_{Q_{2}}\left(i_{2} \circ j_{2} \circ g \circ i_{1} \circ j_{1}\left(q_{1}\right), g \circ i_{1} \circ j_{1}\left(q_{1}\right)\right) \leqslant \sup _{q \in Q_{2}}\left\{d_{Q_{2}}\left(i_{2} \circ j_{2}\left(q_{2}\right), q_{2}\right)\right\} \\
=\delta\left(F_{2} \iota\right) .
\end{gathered}
$$

For the second

$$
\begin{aligned}
d_{Q_{2}}\left(g \circ i_{1} \circ j_{1}\left(q_{1}\right), g\left(q_{1}\right)\right) \leqslant & {\left[\text { because } g \in Q_{1} \rightarrow{ }^{1} Q_{2}\right] } \\
& d_{Q_{1}}\left(i_{1} \circ j_{1}\left(q_{1}\right), q_{1}\right) \\
= & \delta\left(F_{1} \iota\right) .
\end{aligned}
$$

We see

$$
\begin{aligned}
\delta(F \iota) \leqslant & \delta\left(F_{1} \iota\right)+\delta\left(F_{2} \iota\right) \\
\leqslant & {[\text { induction] }} \\
& \left(c\left(F_{1}\right)+c\left(F_{2}\right)\right) \cdot \delta(\iota)
\end{aligned}
$$




$$
=c(F) \cdot \delta(\iota)
$$

Part (d2)

$$
d_{F P \rightarrow}{ }^{e} F Q\left(F \iota, F \iota^{\prime}\right)=\max \left\{d_{F P \rightarrow F Q}\left(F i, F i^{\prime}\right), d_{F Q \rightarrow F P}\left(F j, F j^{\prime}\right)\right\} .
$$

For the first component, we have

$$
d_{F P \rightarrow F Q}\left(F i, F i^{\prime}\right)=\sup _{f \in F P, q \in Q_{1}}\left\{d_{Q_{2}}\left(F i(f)(q), F i^{\prime}(f)(q)\right)\right\} .
$$

Let $f \in F P, q \in Q_{1}$. Then

$$
\begin{aligned}
d_{Q_{2}}\left(F i(f)(q), F i^{\prime}(f)(q)\right)= & d_{Q_{2}}\left(i_{2} \circ f \circ j_{1}(q), i_{2}^{\prime} \circ f^{\prime} j_{1}^{\prime}(q)\right) \\
\leqslant & d_{Q_{2}}\left(i_{2} \circ f \circ j_{1}(q), i_{2}^{\prime} \circ f^{\prime} j_{1}(q)\right)+d_{Q_{2}}\left(i_{2}^{\prime} \circ \circ \circ j_{1}(q), i_{2}^{\prime} \circ f^{\prime} \circ j_{1}(q)\right) \\
\leqslant & d_{P_{2} \rightarrow Q_{2}}\left(i_{2}, i_{2}^{\prime}\right)+d_{Q_{2}}\left(i_{2}^{\prime} \circ f^{\prime} \circ j_{1}(q), i_{2}^{\prime} \circ f^{\prime} j_{1}^{\prime}(q)\right) \\
\leqslant & \text { because } \left.i_{2}^{\prime} \text { is isometric }, f \in P_{1} \rightarrow{ }^{1} P_{2}\right] \\
& d_{P_{2} \rightarrow Q_{2}}\left(i_{2}, i_{2}^{\prime}\right)+d_{Q_{1} \rightarrow P_{1}}\left(j_{1}, j_{1}^{\prime}\right) .
\end{aligned}
$$

(Again, in the case of ultra-metric spaces, we would have "max" here.)

Likewise, we have for the second component

$$
d_{F Q \rightarrow F Q}\left(F j, F j^{\prime}\right) \leqslant d_{P_{1} \rightarrow Q_{1}}\left(i_{1}, i_{1}^{\prime}\right)+d_{Q_{2} \rightarrow P_{2}}\left(j_{2}, j_{2}^{\prime}\right) .
$$

Together this implies

$$
\begin{aligned}
d_{F P \rightarrow} e_{F Q}\left(F \iota, F \iota^{\prime}\right) \leqslant & d_{P_{1} \rightarrow} e_{Q_{1}}\left(F_{1} \iota, F_{1} \iota^{\prime}\right)+d_{P_{2} \rightarrow} e_{Q_{2}}\left(F_{2} \iota, F_{2} \iota^{\prime}\right) \\
\leqslant & {[\text { induction] }} \\
& \left(c\left(F_{1}\right)+c\left(F_{2}\right)\right) \cdot d_{P \rightarrow} e_{Q}\left(\iota, \iota^{\prime}\right) \\
= & c(F) \cdot d_{P \rightarrow} e_{Q}\left(\iota, \iota^{\prime}\right) .
\end{aligned}
$$

Case (f) $F=F_{1} \times F_{2}$ :

Part (f1)

$$
\begin{aligned}
\delta(F \iota) & =d_{F Q \rightarrow F Q}\left(F i \circ F j, i d_{F Q}\right) \\
& =\sup _{\bar{q} \in F Q}\left\{d_{F Q}(F i \circ F j(\bar{q}), \bar{q})\right\} \\
& =\sup _{<q_{1}, q_{2}>\in F Q}\left\{d_{F Q}\left(<i_{1} \circ j_{1}\left(q_{1}\right), i_{2} \circ j_{2}\left(q_{2}\right)>,<q_{1}, q_{2}>\right)\right\} \\
& =\sup _{<q_{1}, q_{2}>\in F Q}\left\{\max \left\{d_{Q_{1}}\left(i_{1} \circ j_{1}\left(q_{1}\right), q_{1}\right), d_{Q_{2}}\left(i_{2} \circ j_{2}\left(q_{2}\right), q_{2}\right)\right\}\right. \\
& =\max \left\{\sup _{q_{1} \in Q_{1}}\left\{d_{Q_{1}}\left(i_{1} \circ j_{1}\left(q_{1}\right), q_{1}\right)\right\}, \sup _{q_{2} \in Q_{2}}\left\{d_{Q_{2}}\left(i_{2} \circ j_{2}\left(q_{2}\right), q_{2}\right)\right\}\right\} \\
& =\max \left\{\delta\left(F_{1} \iota\right), \delta\left(F_{2} \iota\right)\right\}
\end{aligned}
$$




$$
\begin{aligned}
\leqslant & {[\text { induction] }} \\
& \left(c\left(F_{1}\right)+c\left(F_{2}\right)\right) \cdot \delta(\iota) \\
= & c(F) \cdot \delta(\iota) .
\end{aligned}
$$

Part (f2)

$$
\begin{aligned}
& d_{F P \rightarrow} e_{F Q}\left(F i, F i^{\prime}\right)=\sup _{\bar{p} \in F P}\left\{d_{F Q}\left(F i(\bar{p}), F i^{\prime}(\bar{p})\right)\right\} \\
& =\sup _{<p_{1}, p_{2}>\in F P}\left\{d_{F Q}\left(<i_{1}\left(p_{1}\right), i_{2}\left(p_{1}\right)>,<i_{1}^{\prime}\left(p_{2}\right), i_{2}^{\prime}\left(p_{2}\right)>\right)\right\} \\
& =\max \left\{\sup _{p_{1} \in P_{1}}\left\{d_{Q_{1}}\left(i_{1}\left(p_{1}\right), i_{1}^{\prime}\left(p_{1}\right)\right)\right\}, \sup _{p_{2} \in P_{2}}\left\{d_{Q_{2}}\left(i_{2}\left(p_{2}\right), i_{2}^{\prime}\left(p_{2}\right)\right)\right\}\right\} \\
& =\max \left\{d_{P_{1} \rightarrow Q_{1}}\left(i_{1}, i_{1}^{\prime}\right), d_{P_{2} \rightarrow Q_{2}}\left(i_{2}, i_{2}^{\prime}\right)\right\} \text {. }
\end{aligned}
$$

Similarly, we have

$$
d_{F Q \rightarrow F P}\left(F j, F j^{\prime}\right)=\max \left\{d_{Q_{1} \rightarrow P_{1}}\left(j_{1}, j_{1}^{\prime}\right), d_{Q_{2} \rightarrow P_{2}}\left(j_{2}, j_{2}^{\prime}\right)\right\} .
$$

Thus we obtain

$$
\begin{aligned}
d_{F P \rightarrow} e_{F Q}\left(F \iota, F \iota^{\prime}\right)= & \max \left\{d_{P_{1} \rightarrow} e_{Q_{1}}\left(F_{1} \iota, F_{1} \iota^{\prime}\right), d_{Q_{2} \rightarrow} e_{P_{2}}\left(F_{2} \iota, F_{2} \iota^{\prime}\right)\right\} \\
\leqslant & {[\text { induction }] } \\
& \max \left\{c\left(F_{1}\right), c\left(F_{2}\right)\right\} \cdot d_{P \rightarrow} e_{Q}\left(\iota, \iota^{\prime}\right) \\
= & c(F) \cdot d_{P \rightarrow} e_{Q}\left(\iota, \iota^{\prime}\right) .
\end{aligned}
$$

\section{CoRollary 5.5}

For every $F \in F u n c$, with $0 \leqslant c(F)<1$, we have

(1) $F$ is a contracting functor, and

(2) $F$ is a hom-contracting functor.

\section{COROLLARY 5.6}

Every reflexive domain equation over $C$ of the form

$$
P \cong F P,
$$

for which $F \in$ Func and $c(F)<1$, has a unique solution (up to isomorphism). 


\section{ConClusions}

We have presented a technique for constructing fixed points of certain functors over a category of complete metric spaces. This enables us to solve the reflexive domain equations associated with these functors. The technique is an adaptation of the limit construction that was first used in the context of certain partial orders (continuous lattices, complete lattices, complete partial orders). Nevertheless, we have encountered some nice metric phenomena in our metric framework. To begin with, the concept of a converging tower is an analogue to the concept of a Cauchy sequence in a complete metric space, and indeed, both have a limit. Furthermore, a contracting functor on our category of metric spaces is a concept analogous to that of a contracting function on a complete metric space, and both are guaranteed to have a fixed point. If we strengthen our requirements on the functor to include hom-contractivity (also analogous to contractivity of a function), we even know that the fixed point is unique (as is the case with a contracting function). Therefore the whole situation looks very much like Banach's theorem in a category-theoretic disguise.

A few questions remain open, however. We are still looking for a functor that is contracting but not hom-contracting, or even better for a functor that is contracting but has several nonisomorphic fixed points. Another point is what can be said about functors where the argument occurs at the left hand side of a general function space construction (all continuous functions, not just the NDI ones).

In any case, the class of functors (and, thus, domain equations) that we can handle is large enough, so that our technique is a useful tool in the construction of domains for the denotational semantics of concurrent programming languages.

\section{RELATED WORK}

The subject of solving reflexive domain equations is not new. Various solutions of the kind of equations mentioned above already exist. We shall not try to give an extensive and complete bibliography on this matter and confine ourselves to the following remarks.

We mention the work of Scott ([Sc]), who uses inverse limit constructions for solving domain equations. Our method of generalizing metric notions in terms of category-theoretical notions shows a clear analogy to the work D. Lehmann ([Le]) did in the context of partial orderings. Our work is also related to the general method of solving reflexive equations of Smyth and Plotkin ([SP]). In the terminology used there, we show that our category $e$ is $\omega$-complete in the limited sense, that all converging towers have direct limits. Further we show that a certain type of $\omega$ continuous functors (called contracting) has a fixed point. (Without having investigated the precise relationship, we also mention here the anology between their notion of an O-category, and the fact that in our category $e$ the hom-sets are complete metric spaces.) 


\section{REFERENCES}

[ABKR] P. America, J. De Bakker, J. KoK, J. Rutten, A Denotational Semantics of a Parallel Object-Oriented Language, Technical Report (CS-R8626), Centre for Mathematics and Computer Science, Amsterdam, 1986.

[BZ] J.W. DE BAKker, J.I. ZuCKer, Processes and the Denotational Semantics of Concurrency, Information and Control 54 (1982), pp. 70-120.

[Du] J. DugundjI, Topology, Allen and Bacon, Rockleigh, N.J., 1966.

[En] R. Engelking, General Topology, Polish Scientific Publishers, 1977.

[Ha] H. HaHN, Reelle Funktionen, Chelsea, New York, 1948.

[Le] D. Lehmann, Categories for Mathematical Semantics, in: Proc. 17th IEEE Symposium on Foundations of Computer Science, 1976.

[ML] S. MAC LANE, Categories for the Working Mathematician, Graduate Texts in Mathematics 5, Springer-Verlag, 1971.

[Sc] D.S. Scotr, Continuous Lattices, in: Toposes, Algebraic Geometry and Logic, Lecture Notes in Mathematics 274, Springer-Verlag, 1972, pp. 97-136.

[SP] M.B. Smyth, G.D. Plotkin, The Category-Theoretic Solution of Recursive Domain Equations, SIAM J. Comput, Vol. 11, No. 4, 1982, pp.761-783. 